\title{
Discrete flexoelectric polarizations and biaxial subphases with periodicities other than three and four layers in chiral smectic liquid crystals frustrated between ferroelectricity and antiferroelectricity
}

\author{
A. D. L. Chandani, ${ }^{1}$ N. M. Shtykov, ${ }^{1}$ V. P. Panov, ${ }^{1}$ A. V. Emelyanenko, ${ }^{1,2}$ Atsuo Fukuda, ${ }^{1,3}$ and J. K. Vij ${ }^{1, *}$ \\ ${ }^{1}$ Department of Electronic and Electrical Engineering, Trinity College, University of Dublin, Dublin 2, Ireland \\ ${ }^{2}$ Department of Physics, Moscow State University, Moscow 119992, Russia \\ ${ }^{3}$ Department of Environmental Materials Science, Faculty of Engineering, Tokyo Denki University, Tokyo 101-8457, Japan
}

(Received 11 May 2005; published 12 October 2005)

\begin{abstract}
The subphase for the temperature range that lies in between $\mathrm{Sm}-C_{A}^{*}$ and the three-layer Sm- $C_{A}^{*}(1 / 3)$ subphase has been confirmed to exist using the measurements of electric-field-induced birefringence, optical rotation, and the characteristic reflection bands in the antiferroelectric liquid-crystalline compound, 1-trifluoromethylundecyl-4-(4'-dodecyloxybiphenyl-4-yl-carbonyloxy)-3-fluorobenzoate (12BIMF10). The measurements of electric-field-induced birefringence and optical rotatory power are made on 25 - $\mu$ m-thick homeotropic cells, and the characteristic reflection bands are observed in free-standing films of thicknesses ranging from 30 to $50 \mu \mathrm{m}$. Several binary mixtures have been prepared by mixing $(S)$-12BIMF10 with (S)-4-(1-methylheptyloxycarbonyl)-phenyl-4' -octylbiphenyl-4-carboxylate (MHPBC), and the effect of racemization of the compound on the character of the biaxial subphase (other than three and four layers) is also discussed. The results are interpreted in terms of the Emelyanenko-Osipov model [Phys. Rev. E 68, 051703 (2003)]; the effective long-range couplings between the director orientations in separated smectic layers emerge after the minimization of free energy with respect to the total (ordinary spontaneous and discrete flexoelectric) polarizations, lifting the degeneracy and producing the nonplanar structures of the subphases.
\end{abstract}

DOI: 10.1103/PhysRevE.72.041705

PACS number(s): 61.30.Eb, 64.70.Md, 77.80.Bh, 77.84.Nh

\section{INTRODUCTION}

In most of the ordinary liquid-crystalline phases-nematic or chiral nematic, smectic- $A$ (Sm- $A)$, and smectic- $C$ $(\mathrm{Sm}-\mathrm{C})$ - that appear successively on lowering the temperature from the isotropic phase, the symmetry is so high that centrosymmetric distributions around the molecular long axis and head-tail equivalence, due to thermal rotational and other motions, prevent the occurrence of ferroelectricity. In the chiral smectic- $C$ phase $\left(\mathrm{Sm}-C^{*}\right)$, however, the symmetry is low enough to allow the existence of chirality-induced improper ferroelectricity, which does not result from the dipole-dipole interaction. The distribution is now biased in a particular direction and sense and becomes noncentrosymmetric; the low local symmetry forces the molecular transverse dipoles, if there are any, to align at least partially and produces a spontaneous polarization along the $C_{2}$ axis in the middle of each smectic layer given by

$$
\mathbf{P}_{\mathrm{s}}=-\chi c_{\mathrm{s}} \boldsymbol{\xi}
$$

Here $\boldsymbol{\xi}=(\mathbf{n} \cdot \mathbf{e})[\mathbf{n} \times \mathbf{e}]$ is the pseudovector order parameter of $\mathrm{Sm}-C^{*}$, e is normal to the smectic layer, $\chi$ the dielectric susceptibility, and $c_{\mathrm{s}}$ a pseudoscalar determined by molecular chirality. The macroscopic helical structure produces the flexoelectric polarization

\footnotetext{
*Author to whom correspondence should be addressed. Electronic address: jvij@tcd.ie
}

$$
\mathbf{P}_{\mathrm{f}}=-\chi c_{\mathrm{f}} \nabla \times \boldsymbol{\xi}=\chi c_{\mathrm{f}} \boldsymbol{\xi} \frac{\partial \phi}{\partial z}
$$

Since it is parallel to the spontaneous polarization given by Eq. (1), it does not affect the synclinic ferroelectric structure of the phase in a qualitative way [1-3].

The discovery of antiferroelectricity in the chiral smectic$C_{A}$ phase $\left(\mathrm{Sm}-C_{A}^{*}\right)$ that lacks truly long-range positional order was surprising and very remarkable $[4,5]$. So far as the in-layer spontaneous polarizations are concerned, the same mechanism is operative as in $\mathrm{Sm}-C^{*}$ and the spontaneous polarization emerges along the $C_{2}$ axis in the middle of each smectic layer [6-8]. Since the smectic layer order is high in Sm- $C_{A}^{*}[9]$, the discrete model is suitable to use [10] and the $i$ th layer is therefore considered to have the spontaneous polarization

$$
\mathbf{P}_{\mathrm{s}, i}=-\chi c_{\mathrm{s}} \boldsymbol{\xi}_{i}
$$

The sinusoidal density-wave character of the smectic layer may be incompatible with the anticlinic antiferroelectric ordering; it could not be decided in which of the two possible senses the molecules may tilt in the minimum-density region. On the other hand, it can naturally be considered that each layer, rather clearly defined, has its own polarization. Because of its anticlinic ordering, Eq. (3) changes sign from layer to layer and hence the net spontaneous polarization becomes zero as the term "antiferroelectricity" implies.

The existence of some Sm- $C^{*}$-like phases was noticed by Fukui et al. during their detailed differential scanning calorimetry (DSC) studies of 4-(1-methylheptyloxycarbonyl) phenyl-4' -octyloxybiphenyl-4-carboxylate (MHPOBC) [11]. 
Chandani et al. designated these phases as $\mathrm{Sm}-C_{\alpha}^{*}, \mathrm{Sm}-C_{\beta}^{*}$, and Sm- $C_{y}^{*}$ in the order of decreasing temperature, identifying Sm- $C_{\beta}^{*}$ as ordinary synclinic Sm- $C^{*}[4,5]$ while discovering an anticlinic antiferroelectric $\mathrm{Sm}-C_{A}^{*}$ phase. Gorecka et $a l$. soon proved that $\mathrm{Sm}-C_{\gamma}^{*}$ is a ferrielectric phase [12], and Takezoe et al. proposed the three-layer Ising model with one ferroelectric and two antiferroelectric orderings within a single period [13]. Furthermore, Okabe et al. found another antiferroelectric phase other than $\mathrm{Sm}-C_{A}^{*}$ in MHPBC, designating it as $\mathrm{AF}$ [14], and Isozaki et al. proposed the fourlayer Ising model, where the tilt sense changes every two layers and the repeating unit consists of four layers with two ferroelectric and two antiferroelectric orderings [15]. Because of the small free-energy difference between Sm- $C_{A}^{*}$ and $\mathrm{Sm}-C^{*}$ together with the low barrier in between them due to the azimuthal angle freedom, the system is frustrated between synclinic ferroelectric and anticlinic antiferroelectric orderings. The frustration causes the temperatureinduced sequence of phase transitions. The polar smectic phases thus produced are often called "subphases" in contrast to the fundamental phases $\mathrm{Sm}-C_{A}^{*}, \mathrm{Sm}-C^{*}$, and $\mathrm{Sm}-A$. By emphasizing the existence of biaxial subphases with periodicities other than three and four layers, Isozaki et al. suggested that the biaxial subphases may constitute a devil's staircase, which can be described by the one-dimensional Ising model with long-range repulsive interactions. The sense of tilting direction in a smectic layer can be specified by an Ising-like variable that represents a collective property; then the long-range interaction can be interpreted as a coupling between the director orientations in distant smectic layers. They tried to specify the biaxial subphase by the fraction of $q_{T}=[F] /([A]+[F])$. Here $[F]$ and $[A]$ are the ferroelectric and antiferroelectric orderings. Since all of these biaxial subphases are closely related with $\mathrm{Sm}-C_{A}^{*}$, Isozaki et al. designated them as $\mathrm{Sm}-C_{A}^{*}\left(q_{T}\right)$ 's [15-17]. Thus $\mathrm{Sm}-C_{A}^{*}(1 / 3)$ and $\mathrm{Sm}-C_{A}^{*}(1 / 2)$ are the designations for $\mathrm{Sm}-C_{\gamma}^{*}$ and $\mathrm{AF}$, respectively. It should be noted that $q_{T}=0$ in $\operatorname{Sm}-C_{A}^{*}$ and $q_{T}=1$ in Sm- $C^{*}$.

The biaxial subphases are much more interesting phases than the fundamental phases $\mathrm{Sm}-C_{A}^{*}$ and $\mathrm{Sm}-C^{*}$ and scientifically more intriguing. A discrete flexoelectric polarization given by

$$
\mathbf{P}_{\mathrm{f}, i}=-\chi c_{\mathrm{f}} \cos \theta \Delta \mathbf{n}_{i \pm 1}=-\chi c_{\mathrm{f}} \cos \theta\left(\mathbf{n}_{i+1}-\mathbf{n}_{i-1}\right),
$$

where $\mathbf{n}_{i}=\left(\sin \theta \cos \phi_{i}, \sin \theta \sin \phi_{i}, \cos \theta\right)$, may emerge within each layer and play an important role in determining the detailed orientational structure of the subphase [18-23]. The real issue is the fact that the discrete flexoelectric polarization may not be parallel to the ordinary spontaneous polarization given by Eq. (3). This is acceptable since the point symmetry of a layer is so reduced that the $C_{2}$ axis may generally disappear in biaxial subphases. Figure 1 illustrates how the in-layer polarizations emerge in $\mathrm{Sm}-C_{A}^{*}(1 / 3)$ and $\mathrm{Sm}-C_{A}^{*}(1 / 2)$ by using the Ising model for the sake of simplicity. The ordinary in-layer spontaneous polarizations always appear perpendicularly to the tilt plane and have the same magnitude with the sign determined by the tilt sense. The in-layer discrete flexoelectric polarizations are, on the
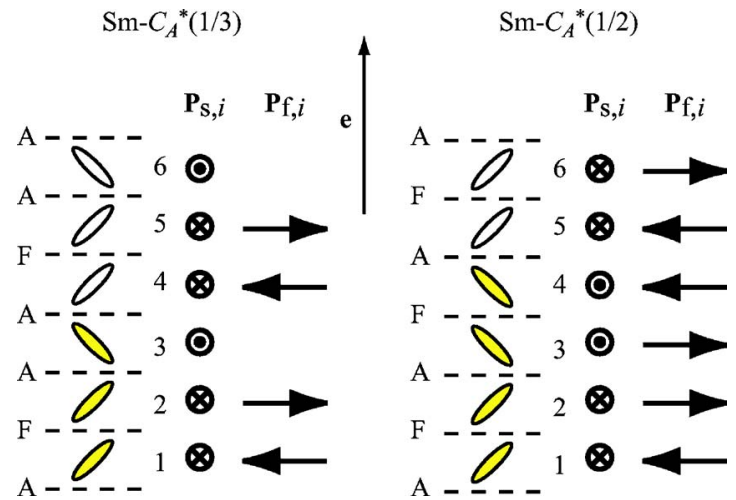

FIG. 1. (Color online) The emergence of the in-layer polarizations is indicated. The spontaneous polarization $\mathbf{P}_{\mathrm{s}, i}$ and the discrete flexoelectric polarization $\mathbf{P}_{\mathrm{f}, i}$ are illustrated using the Ising model. Note that $\mathbf{P}_{\mathrm{f}, i}$ appears in the tilt plane while $\mathbf{P}_{\mathrm{s}, i}$ is perpendicular to it. The unit cell is shown as shaded (yellow colored).

other hand, parallel to the tilt plane. They may have the same magnitude with the sign determined by $\Delta \mathbf{n}_{i \pm 1}$. They may become zero when the tilt senses in adjacent layers are the same-i.e., $\Delta \mathbf{n}_{i \pm 1}=0$ as seen in the third layer in $\mathrm{Sm}-C_{A}^{*}(1 / 3) ; \mathbf{P}_{\mathrm{f}, i}=0$ for $i=3$ is consistent with the presence of the $C_{2}$ axis perpendicularly to the tilt plane in the middle of this layer.

The emergence of two kinds of polarizations in a smectic layer which are not parallel to each other brings about two mutually related important consequences: (i) By taking the polarization-dependent short-range (nearest neighbor) interactions into account only, the effective long-range couplings between the director orientations in separated smectic layers emerge after the minimization of free energy with respect to the polarization. (ii) In spite of the fact that almost all intermolecular interactions favor the planar structure, the azimuthal angle difference between the directors in adjacent layers in the biaxial subphase may be considerably deviated from either of $\Delta \phi=0$ (synclinic) or $\Delta \phi=\pi$ (anticlinic). Foreseeing these consequences, Emelyanenko and Osipov showed that a simple discrete model can be used to describe the entire sequence of biaxial subphases with increasing $q_{T}$ as the temperature rises and to determine the nonplanar structure of each subphase without additional assumptions $[20,21]$. In fact, $\mathrm{Sm}-C_{A}^{*}(1 / 3)$ and $\mathrm{Sm}-C_{A}^{*}(1 / 2)$ have nonplanar structures. These have now been confirmed through a number of experiments using different sophisticated techniques [24-36]. Each subphase is characterized by a unique set of azimuthal angles $\left\{\phi_{i}\right\}$, which specifies different azimuthal orientations of the director in each smectic layer within the unit crystallographic cell and has the chirality determined by the sign of $c_{\mathrm{s}} c_{\mathrm{f}}$ in Eqs. (3) and (4). In other words, the biaxial subphase $\mathrm{Sm}-C_{A}^{*}\left(q_{T}\right)$ has a microscopic (short-pitch) helical structure, though highly distorted, and the pitch is given by $2 /\left(1-q_{T}\right)$ in the unit of the number of smectic layers. Emelyanenko and Osipov replaced the Ising model with a more realistic planar rotator model and considered the total free energy of a subphase, 


$$
F=\sum_{i=1}^{N}\left\{F_{i}+\Delta F_{i}\right\} .
$$

Here $N$ is the total number of smectic layers and $F_{i}$ does not depend on the polarization but depends only on the tilt angle and the director orientations between successive layers $[20,21]$. All polarization-dependent terms are included in $\Delta F_{i}$, which can be written as

$$
\begin{aligned}
\Delta F_{i}= & \frac{1}{2 \chi}\left\{\mathbf{P}_{i}^{2}+g\left(\mathbf{P}_{i} \cdot \mathbf{P}_{i+1}+\mathbf{P}_{i} \cdot \mathbf{P}_{i-1}\right)\right\}+c_{\mathrm{s}}\left(\mathbf{P}_{i} \cdot \boldsymbol{\xi}_{i}\right) \\
& +c_{\mathrm{f}} \cos \theta\left(\mathbf{P}_{i} \cdot \Delta \mathbf{n}_{i \pm 1}\right),
\end{aligned}
$$

where $\chi$ is the dielectric susceptibility and $g$ is the dimensionless parameter that characterizes the relative strength of the coupling of polarizations in adjacent layers and is less than unity.

Several research groups have investigated $\mathrm{Sm}-C_{A}^{*}(1 / 3)$ and $\mathrm{Sm}-C_{A}^{*}(1 / 2)$ in various compounds and mixtures; their frequent emergence and nonplanar structures have been well confirmed experimentally [24-36]. On the contrary, a few groups have reported the sequential emergence of biaxial subphases other than $\mathrm{Sm}-C_{A}^{*}(1 / 3)$ and $\mathrm{Sm}-C_{A}^{*}(1 / 2)$ with emphasis on the staircase character, which the EmelyanenkoOsipov theory naturally predicts as a result of the effective long-range couplings among the director orientations in distant smectic layers. In recent publications on the subphases, resulting from the frustration between synclinic ferroelectric $\mathrm{Sm}-C^{*}$ and anticlinic antiferroelectric $\mathrm{Sm}-C_{A}^{*}$, the staircase character has been intentionally or unintentionally disregarded. It seems to have possibly been assumed that the apparent coexistence of Sm- $C_{A}^{*}$ and $\mathrm{Sm}-C_{A}^{*}(1 / 3), \operatorname{Sm}-C_{A}^{*}(1 / 3)$ and $\mathrm{Sm}-C_{A}^{*}(1 / 2)$, or $\mathrm{Sm}-C_{A}^{*}(1 / 2)$ and $\mathrm{Sm}-C^{*}$ might be the reason for the existence of additional subphases; note that the phase transition between the two successive subphases is of first order. We will later show that this indeed is a misidentification of the additional subphase. The aim of this paper is to provide unambiguous experimental evidence for the emergence of an additional subphase between $\mathrm{Sm}-C_{A}^{*}$ and $\mathrm{Sm}-C_{A}^{*}(1 / 3)$. The experimental proof surely fosters the Emelyanenko-Osipov model for the emergence of a variety of interesting polar phases that are possible to exist due to the frustration between ferroelectricity and antiferroelectricity.

The paper is organized as follows. Section II gives the structural formulas of the two compounds used, MHPBC and 12BIMF10. The section describes the setup for the measurements of the optical rotatry power (ORP), which is a slight modification of the setup for the measurements of the electric-field-induced birefringence [21], and gives the relevant theoretical basis for the technique. Section III consists of two subsections, III A and III B, which include the experimental results. Recently we have shown that the technique for the measurement of the electric-field-induced birefringence in homeotropic aligned cells using a photoelastic modulator (PEM) is highly reliable and a powerful technique for proving the existence of the two kinds of optically uniaxial subphases $\mathrm{Sm}-C_{\alpha, \mathrm{i}}^{*}$ and $\mathrm{Sm}-C_{\alpha, \mathrm{d}}^{*}[21,37]$. In $\mathrm{Sec}$.

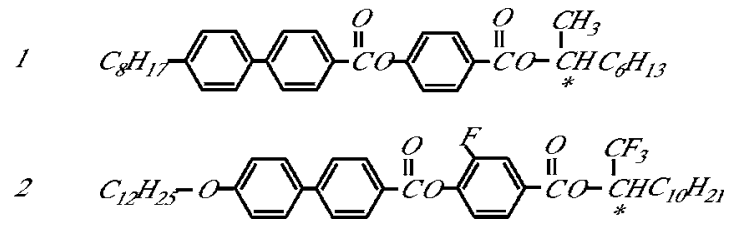

FIG. 2. Chemical structures of the compounds used: $(1)(R)$ - and $(S)$-MHPBC's and (2) $(S)$-12BIMF10.

III A, we first show that the birefringence contours in the $E-T$ (electric-field-temperature) phase diagram are so characteristic that the typical uniaxial and biaxial subphases $\mathrm{Sm}-C_{\alpha}^{*}, \mathrm{Sm}-C_{A}^{*}(1 / 3)$, and $\mathrm{Sm}-C_{A}^{*}(1 / 2)$, as well as the fundamental phases Sm- $A, \mathrm{Sm}-C^{*}$, and $\mathrm{Sm}-C_{A}^{*}$, are easily identifiable. Hence we can establish the gross phase sequence in the binary system of 12BIMF10 and MHPBC as well as that of $(R)$ - and $(S)$-MHPBC's. At the same time, we notice that an unambiguous proof for the existence of additional subphases, other than the typical ones, is not easy even if we also measure ORP. Consequently, in Sec. III B, we try to directly observe the Bragg reflection color and spectra of thick freestanding films due to the macroscopic (long-pitch) helical structure and to correlate them with the $E-T$ phase diagram and the ORP temperature variation. The various experimental results are shown to present an unambiguous evidence for the existence of the new subphase. Discussions are made in Sec. IV about the possible structure of this subphase and these are considered in the framework of the EmeyanenkoOsipov model, which includes competition between the inlayer ferroelectric polarizations and flexoelectric polarizations. The essential theoretical basis of the molecular model by Emelyanenko and Osipov is examined, whereas the unrealistic physical basis of both Ising and clock $(X-Y)$ models is pointed out. Section V concludes the paper.

\section{EXPERIMENT}

The chemical structures of the two compounds used in this study are shown in Fig. 2. Investigations were carried out in nominally pure $(S)$-1-trifluoromethylundecyl-4(4'-dodecyloxybiphenyl-4-yl-carbonyloxy)-3-fluorobenzoate (12BIMF10), $(R)$ - and (S)-4-(1-methylheptyloxycarbonyl)phenyl-4'-octylbiphenyl-4-carboxylate (MHPBC), and binary mixtures of $(S)$-12BIMF10 and $(S)$-MHPBC. The compound 12BIMF10 has a short helical pitch whereas MHPBC has a relatively long helical pitch in $\mathrm{Sm}-C_{A}^{*}[14,17]$. Furthermore, $(S)$-12BIMF10 and $(S)$-MHPBC have opposite helical senses. A free-standing film of the sample, ranging from 30 to $50 \mu \mathrm{m}$ thickness, was drawn on a hole of $1 \mathrm{~mm}$ diameter made in a steel plate of $300 \mu \mathrm{m}$ thick. The film was mounted on a hot stage connected to a temperature controller that controls the temperature of the hot stage within an accuracy of $\pm 0.01{ }^{\circ} \mathrm{C}$. The textural changes with temperature were observed under a polarizing microscope. The selective reflection band of the film was measured at an oblique incidence angle of $20^{\circ}$ using a UV/visible/near-IR spectrophotometer (Perkin Elmer, Lambda 900). A homeotropic cell, used for measuring the electric-field-induced birefringence 


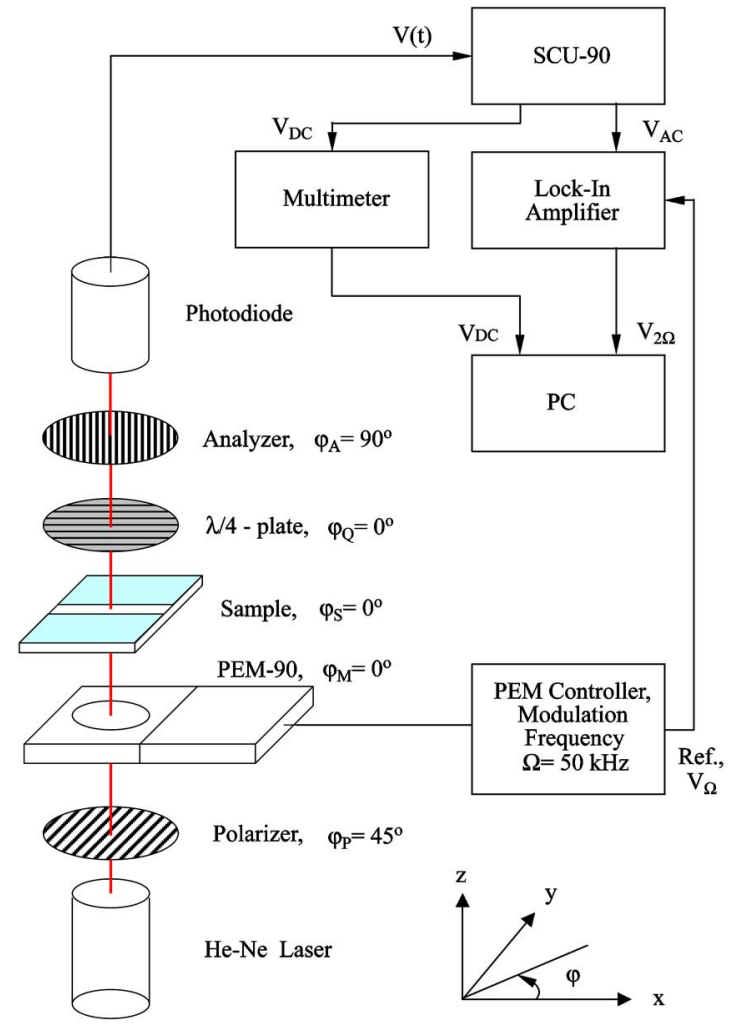

FIG. 3. (Color online) Setup for the measurement of optical rotatory power (ORP). SCU-90 is a signal-conditioning unit used for signal processing including amplification. The electric field when applied is across the direction $y$.

and the optical rotatory power, consists of two glass plates separated by Mylar thin-film spacers of $25 \mu \mathrm{m}$ thickness. The bottom plate has two ITO (indium tin oxide) electrodes for applying an in-plane electric field. The gap between electrodes is about $180 \mu \mathrm{m}$. For aligning the sample homeotropically, both inside surfaces of the glass plates were coated with carboxylato chromium complexes (chromolane), cured at a temperature of $150{ }^{\circ} \mathrm{C}$ for $30 \mathrm{~min}$. The cell was heated and filled with the sample in the isotropic phase and cooled slowly to the $\mathrm{Sm}-A$ phase. It was mounted in the same hot stage that was also used for the free-standing film. The homeotropic alignment was confirmed under the polarizing microscope.

The ORP was measured by using a PEM as schematically illustrated in Fig. 3, which is a slightly modified setup used for obtaining the electric-field-induced birefringence [21]. A $\mathrm{He}-\mathrm{Ne}$ laser with a wavelength of $632.8 \mathrm{~nm}$ and a continuous-wave (cw) output power of about $2 \mathrm{~mW}$ was used as the light source. A signal-conditioning unit (SCU-90) amplifies the photodiode signal and then separates it into dc $\left(V_{\mathrm{DC}}\right)$ and ac $\left(V_{\mathrm{AC}}\right)$ voltages. A digital multimeter and a lock-in amplifier measured the dc voltage $\left(V_{\mathrm{DC}}\right)$ and the rms voltage of the second harmonic of the ac signal $\left(V_{2 \Omega}\right)$, respectively. The right-handed reference frame $(x, y$, and $z)$ was so chosen that the $z$ axis is perpendicular to the smectic layer and the electric field (if it was needed) was applied along the $y$ axis. By using the Jones matrix method, let us calculate the state of polarization of the light after passing through the optical system. The axes of the polarizer $(\mathrm{P})$, PEM (M), the sample (S), the quarter wave plate $(\mathrm{QW})$, and the analyzer (A) make angles $\varphi_{\mathrm{P}}=45^{\circ}, \varphi_{\mathrm{M}}=0^{\circ}, \varphi_{\mathrm{S}}=0^{\circ}$, $\varphi_{\mathrm{QW}}=0^{\circ}$, and $\varphi_{\mathrm{A}}=90^{\circ}$ with respect to the $x$ axis, respectively. The corresponding Jones matrices for these optical components are

$$
\begin{aligned}
& P=\frac{1}{2}\left(\begin{array}{ll}
1 & 1 \\
1 & 1
\end{array}\right), \quad M=\left(\begin{array}{cc}
\exp \left(\mathrm{i} \Phi_{M}\right) & 0 \\
0 & 1
\end{array}\right), \\
& S=\left(\begin{array}{cc}
\cos \alpha & -\sin \alpha \\
\sin \alpha & \cos \alpha
\end{array}\right), \quad Q=\left(\begin{array}{ll}
\mathrm{i} & 0 \\
0 & 1
\end{array}\right),
\end{aligned}
$$

and

$$
A=\left(\begin{array}{ll}
0 & 0 \\
0 & 1
\end{array}\right)
$$

Here $\Phi_{\mathrm{M}}=A_{0} \cos (\Omega t)$ is the PEM retardation and $\alpha$ the optical rotation angle of the sample. The electrical vector of the light wave passed through the optical system, $\mathbf{E}_{\mathrm{T}}$, is expressed by

$$
\begin{aligned}
\mathbf{E}_{\mathrm{T}}= & \frac{1}{2}\left(\begin{array}{ll}
0 & 0 \\
0 & 1
\end{array}\right)\left(\begin{array}{ll}
\mathrm{i} & 0 \\
0 & 1
\end{array}\right)\left(\begin{array}{cc}
\cos \alpha & -\sin \alpha \\
\sin \alpha & \cos \alpha
\end{array}\right)\left(\begin{array}{cc}
\exp \left(\mathrm{i} \Phi_{M}\right) & 0 \\
0 & 1
\end{array}\right) \\
& \times\left(\begin{array}{ll}
1 & 1 \\
1 & 1
\end{array}\right) \mathbf{E}_{\mathrm{I}} \\
= & \frac{1}{2}\left[\cos \alpha+\sin \alpha \exp \left(\mathrm{i} \Phi_{M}\right)\right]\left(\begin{array}{ll}
0 & 0 \\
1 & 1
\end{array}\right) \mathbf{E}_{\mathrm{I}}
\end{aligned}
$$

where $\mathbf{E}_{\mathrm{I}}$ is the electrical vector of the incident light wave. The intensity of the transmitted light is given by

$$
\begin{aligned}
I & =\mathbf{E}_{\mathrm{T}}{ }^{*} \cdot \mathbf{E}_{\mathrm{T}} \\
& =\frac{I_{0}}{4}\left[1+\sin 2 \alpha \cos \Phi_{M}\right] \\
& =\frac{I_{0}}{4}\left[1+\sin 2 \alpha \cos \left(A_{0} \cos \Omega t\right)\right] \\
& =\frac{I_{0}}{4}\left[1+\sin 2 \alpha J_{0}\left(A_{0}\right)-2 \sin 2 \alpha J_{2}\left(A_{0}\right) \cos 2 \Omega t\right],
\end{aligned}
$$

where $I_{0}=\mathbf{E}_{\mathrm{I}}^{*} \cdot \mathbf{E}_{\mathrm{I}}$ is the intensity of the incident light. $J_{0}\left(A_{0}\right)$ and $J_{2}\left(A_{0}\right)$ are the Bessel functions. By adjusting the modulation amplitude to obtain $J_{0}\left(A_{0}\right)=0$, i.e., $A_{0}=2.405$, the formula for the angle of the optical rotation can be written as

$$
\alpha=-\frac{1}{2} \sin ^{-1}\left(\frac{V_{2 \Omega}}{\sqrt{2} J_{2}\left(A_{0}\right) V_{D C}}\right),
$$

where $J_{2}\left(A_{0}\right)=0.432$. The sensitivity of the optical rotatory power measured was better than $4.0 \times 10^{-5} \mathrm{deg}$. 
(a)

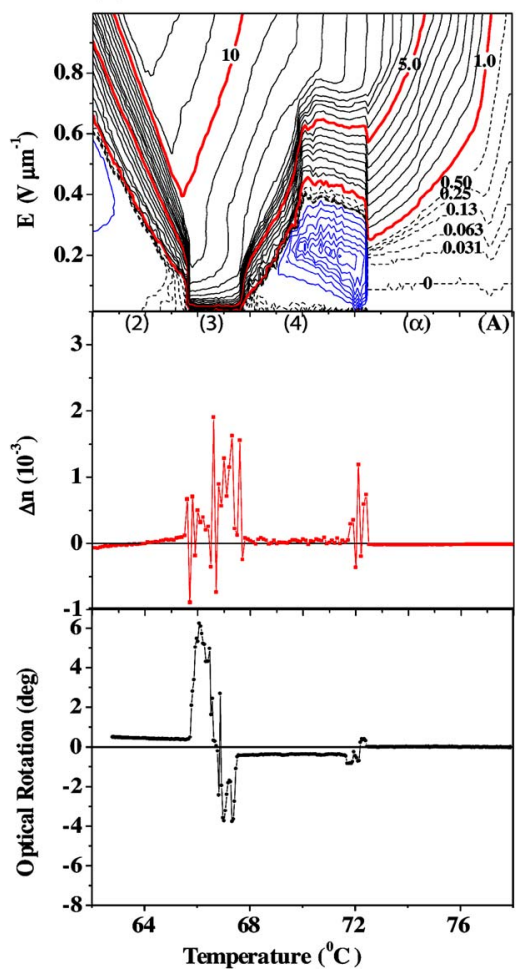

(b)

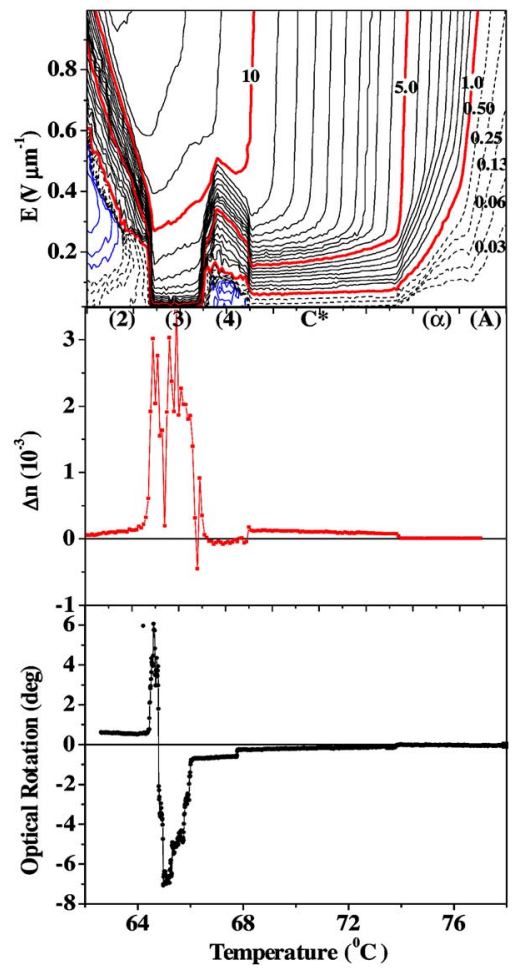

FIG. 4. (Color online) Electric-field-induced birefringence observed during the cooling cycle in 25- $\mu \mathrm{m}$-thick homeotropic cells of (a) nominally pure $(S)$-MHPBC and (b) partially racemized one with $R / S=93 / 7$ in weight. The residual birefringence and optical rotation are given in the lower part; these were measured before applying any electric field. The birefringence contours drawn in solid lines are at steps of $0.5 \times 10^{-3}$ and some auxiliary lines in smaller steps are drawn as dotted lines. The numbers within brackets in (a) and (b) indicate the number of layers in a unit cell. $\alpha, C$, and $A$ are $\mathrm{Sm}-C_{\alpha}^{*}, \mathrm{Sm}-C^{*}$, and $\mathrm{Sm}-A$, respectively. Birefringence contours shown as solid blue lines denote the unwinding of the macroscopic helices in $\mathrm{Sm}-C_{A}^{*}$ and $\mathrm{Sm}-C_{A}^{*}(1 / 2)$.

\section{RESULTS}

\section{A. Characteristic patterns of the birefringence contours in the $E-T$ space}

To identify the three typical subphases $\mathrm{Sm}-C_{A}^{*}(1 / 3)$, $\mathrm{Sm}-C_{A}^{*}(1 / 2)$, and $\mathrm{Sm}-C_{\alpha}^{*}$, Isozaki et al. [38] earlier studied the electric field dependence of conoscopic figures. Later, several groups have used mainly the techniques of dielectric spectroscopy and/or the ORP for the identification and characterization. These methods are relatively convenient and reliable for making the subphase assignments, but still cannot exhibit their characteristic salient features at a glance. In previous papers [21,37], we showed that PEM-based electricfield-induced birefringence measurements in homeotropically aligned cells are fully informative and useful in clarifying the details of the $\mathrm{Sm}-C_{\alpha}^{*}$ subphase; the birefringence contours in the $E-T$ (electric-field-temperature) phase diagram showed characteristic patterns which revealed the existence of the two kinds of the uniaxial Sm- $C_{\alpha}^{*}$ subphases, $\mathrm{Sm}-C_{\alpha, \mathrm{i}}^{*}$ and $\mathrm{Sm}-C_{\alpha, \mathrm{d}}^{*}$. Similarly, we can determine the characteristic features of $\mathrm{Sm}-C_{A}^{*}(1 / 3)$ and $\mathrm{Sm}-C_{A}^{*}(1 / 2)$ as well as $\mathrm{Sm}-C_{A}^{*}, \mathrm{Sm}-C^{*}$, and $\mathrm{Sm}-C_{\alpha}^{*}$ as illustrated in Figs. 4(a) and 4(b). Isozaki et al. earlier reported that the gross phase sequence of nominally optically pure $(S)$ - or $(R)$-MHPBC is $\mathrm{Sm}-C_{A}^{*}-\mathrm{Sm}-C_{A}^{*}(1 / 3)-\mathrm{Sm}-C_{A}^{*}(1 / 2)-\mathrm{Sm}-C_{\alpha}^{*}$ and that of partially racemized MHPBC with a ratio of $R / S=93 / 7$ in weight is $\mathrm{Sm}-C_{A}^{*}-\mathrm{Sm}-C_{A}^{*}(1 / 3)-\mathrm{Sm}-C_{A}^{*}(1 / 2)-\mathrm{Sm}-C^{*}-\mathrm{Sm}-C_{\alpha}^{*}$, respectively [38]. These phase sequences are easily identified on the temperature $(E=0)$ axis in Fig. 4. The residual birefringence and ORP shown in the lower part also reflects the phase sequence as well as the textural variations. The small variations in the optical rotation and the residual birefrin- gence at $72{ }^{\circ} \mathrm{C}$ shown in Fig. 4(a) are possibly due to the presence of another subphase. This will be explained in detail later.

Much more evidently, each phase shows its characteristic pattern of the birefringence contours. When the applied electric field is sufficiently high, all the phases under consideration may become completely unwound $\mathrm{Sm}-C^{*}$. In fact, birefringence contours are almost parallel to the field $E$ axis as obviously seen in Fig. 4(b). This means that the electroclinic effect is small in completely unwound $\mathrm{Sm}-C^{*}$ and hence that $\theta$ is determined mainly by $T$ and almost independent of $E$. On the contrary, the electroclinic effect plays an important role in $\mathrm{Sm}-A$, and the birefringence contours are actually oblique as seen in Figs. 4(a) and 4(b). Because of the unwinding process of the short-pitch helical structure in Sm- $C_{\alpha}^{*}$, the threshold field for the phase transition into $\mathrm{Sm}-C^{*}$ is relatively high but becomes lower with falling temperature as the short pitch becomes longer. In the temperature region just below $\mathrm{Sm}-A$, some dents or valleys are observed in the birefringence contour lines, and the extrapolation to the zero field indicates the phase transition between $\mathrm{Sm}-A$ and $\mathrm{Sm}-C_{\alpha}^{*}$ at $E=0$, as already pointed out in our previous paper [21]. The unwinding process of the ordinary macroscopic (long-pitch) helical structure of the antiferroelectric phases $\mathrm{Sm}-C_{A}^{*}$ and $\mathrm{Sm}-C_{A}^{*}(1 / 2)$ contrasts with the ferrielectric and ferroelectric phases $\operatorname{Sm}-C_{A}^{*}(1 / 3)$ and $\mathrm{Sm}-C^{*}$ as is revealed by Fig. 4(a). Within each phase, the threshold field for the field-induced phase transition into $\mathrm{Sm}-C^{*}$ becomes higher with increasing the in-layer director tilt angle $\theta$ as the temperature $T$ falls.

The field-induced birefringence apparently always increases during the unwinding process in $\operatorname{Sm}-C_{A}^{*}(1 / 3)$ and $\mathrm{Sm}-C^{*}$. In particular, a very small electric field induces a 
large birefringence in $\mathrm{Sm}-C_{A}^{*}(1 / 3)$. This results from the fact that $\mathrm{Sm}-C_{A}^{*}(1 / 3)$ is a ferrielectric phase and has the spontaneous polarization and, more importantly, that its macroscopic helical structure has a very long, sometimes infinite, pitch. The completely unwound nonplanar structure of $\mathrm{Sm}-C_{A}^{*}(1 / 3)$, which has three-smectic-layer periodicity, appears to exist stably in Fig. 4(a), since the birefringence contours become nearly vertical at around $E$ $=0.15-0.35 \mathrm{~V} \mathrm{\mu m} \mathrm{m}^{-1}$. This is not the case in Fig. 4(b), and racemization seems to make the unwound nonplanar structure unstable in $R / S=93 / 7$ MHPBC. The completely unwound planar structure of Sm- $C^{*}$ can also be easily attained at around $E=0.25-0.35 \mathrm{~V} \mathrm{\mu m}^{-1}$ in Fig. 4(b), and then the birefringence contours become vertical as already pointed out above. In Sm- $C_{A}^{*}$ and Sm- $C_{A}^{*}(1 / 2)$, on the other hand, the in-layer directors first tend to become parallel to the applied field during the unwinding process; consequently, the fieldinduced birefringence once becomes negative and the birefringence contours may form the negative peaks [described by closed (blue) lines] as clearly seen at around $E$ $=0.2 \mathrm{~V} \mu \mathrm{m}^{-1}$ in the temperature range of $\mathrm{Sm}-C_{A}^{*}(1 / 2)$, $67.8-72.5^{\circ} \mathrm{C}$, in Fig. 4(a). The distinction of $\mathrm{Sm}-C_{A}^{*}(1 / 2)$ from $\mathrm{Sm}-C_{A}^{*}$ can easily be made by relatively small critical fields for the unwinding and for the phase transition to $\mathrm{Sm}-C_{A}^{*}(1 / 3)$ or $\mathrm{Sm}-C^{*}$. Two different responses are seen in $\mathrm{Sm}-C_{A}^{*}(1 / 2)$ under the electric field. In other words, the $\mathrm{Sm}-C_{A}^{*}(1 / 2)$ temperature range is divided into two: the fieldinduced phase transition occurs directly to unwound $\mathrm{Sm}-\mathrm{C}^{*}$ on the high-temperature side, $69.8-72.5{ }^{\circ} \mathrm{C}$ in Fig. 4(a) and $65.5-66.9^{\circ} \mathrm{C}$ in Fig. 4(b), while it takes place via $\mathrm{Sm}-C_{A}^{*}(1 / 3)$, although considerably deformed by the applied field, on the low-temperature side, $67.8-69.8^{\circ} \mathrm{C}$ in Fig. 4(a) and $64.8-65.5^{\circ} \mathrm{C}$ in Fig. 4(b).

Isozaki et al. reported additional subphases $\mathrm{FI}_{L}$ and $\mathrm{FI}_{H}$ below and above $\mathrm{Sm}-C_{A}^{*}(1 / 3)$, which are to be appropriately designated as $\mathrm{Sm}-C_{A}^{*}\left(0<q_{T}<1 / 3\right)$ and $\mathrm{Sm}-C_{A}^{*}\left(1 / 3<q_{T}\right.$ $<1 / 2)[15,16,38]$. Even the existence of $\mathrm{Sm}-C_{A}^{*}\left(1 / 2<q_{T}\right.$ $<1$ ) has been alluded to by a few groups [39-42]. Therefore, we tried to identify some of these additional subphases in the $E-T$ phase diagrams mapped with birefringence contours shown in Figs. 4(a) and 4(b). Unfortunately, however, we could not confirm the existence of any such subphases. The temperature range of $\mathrm{Sm}-C_{A}^{*}\left(0<q_{T}<1 / 3\right)$ seems to be as narrow as $10 \mathrm{mK}\left(0.01{ }^{\circ} \mathrm{C}\right)$ or less in the pure and partially racemized MHPBC here studied. According to the previous data published by Isozaki et al., $\operatorname{Sm}-C_{A}^{*}\left(1 / 3<q_{T}<1 / 2\right)$ has the temperature range as wide as $50 \mathrm{mK}\left(0.05^{\circ} \mathrm{C}\right)$ or more [38], but it could not be detected by the PEM method with a temperature step of $10 \mathrm{mK}\left(0.01{ }^{\circ} \mathrm{C}\right)$, either. The aforementioned division of the $\mathrm{Sm}-C_{A}^{*}(1 / 2)$ temperature region into two parts, low and high, might possibly disturb the conoscopic identification method used by Isozaki et al. In fact, they apparently identified the low-temperature region as Sm- $C_{A}^{*}\left(1 / 3<q_{T}<1 / 2\right)$, noticing the further subdivision of the high-temperature region; the contours just below the field-induced phase transition into $\mathrm{Sm}-\mathrm{C}^{*}$ behave differently so that these lines increase with temperature on the left side $\left(69.8-70.3{ }^{\circ} \mathrm{C}\right)$ while decrease on the right side $\left(70.3-72.5^{\circ} \mathrm{C}\right)$ as clearly seen in Fig. 4(a). Regarding Sm- $C_{A}^{*}\left(1 / 2<q_{T}<1\right)$, we have observed some peculiar domain formation in nominally pure MHPBC during the cooling process under a polarizing microscope. This domain formation is also seen in the $E-T$ phase diagram as disturbed birefringence contours at low electric fields as well as in the residual birefringence and ORP data given in Fig. 4(a) just below $\mathrm{Sm}-C_{\alpha}^{*}$. Although we have not yet clarified the real cause of the domain formation, we would like to point out that Hatano et al. and Panarin et al. also observed possible $\mathrm{Sm}-C_{A}^{*}\left(1 / 2<q_{T}<1\right)$ after some thermal treatments $[39,41,42]$. It should also be noted that the divergence of pitch and the change of handedness occur in $\mathrm{Sm}-C_{A}^{*}(1 / 3)$ phase in both nominally pure and partially racemized $\mathrm{MH}-$ PBC as shown at the bottom of Fig. 4(a) and 4(b).

In this way, we have to be very careful in proving the existence of additional biaxial subphases other than $\mathrm{Sm}-C_{A}^{*}(1 / 3)$ and $\mathrm{Sm}-C_{A}^{*}(1 / 2)$. At least two independent methods need to be adopted. Directly observing the Bragg reflection color due to the helical structure, if possible, must be the most persuasive way to prove the existence of the additional subphases. In fact, Itoh et al. reported that $\mathrm{Sm}$ $-C_{A}^{*}\left(0<q_{T}<1 / 3\right)$ in nominally pure $(R)$-12BIMF10 shows such red color [40]. In order to figure out the gross phase sequence, which was obscured at that time, we first studied the $E-T$ phase diagrams of the binary mixture system of nominally pure $(S)$-12BIMF10 and nominally pure $(S)$-MHPBC. The results are summarized in Figs. 5(a)-5(c). In $(S)$-12BIMF10 given in (a), $\mathrm{Sm}-C_{A}^{*}(1 / 3)$ does not exist stably at $E=0$ and the gross phase sequence must be $\mathrm{Sm}-C_{A^{*}}-\mathrm{Sm}-C_{\alpha}^{*}-\mathrm{Sm} A$. The $\mathrm{Sm}-C_{A}^{*}(1 / 3)$ subphase stabilizes gradually as the ratio of $(S)$-MHPBC increases and becomes to exist even at $E=0$ as is clear in (c). By further increasing the $(S)$-MHPBC ratio, $S m-C_{A}^{*}(1 / 2)$ now becomes stabilized though not shown in Fig. 5. Such an evolution of the subphase sequences as also observed in Figs. 4(a) and 4(b) are quite common and have been observed in many binary mixture systems $[15,16,38]$.

\section{B. Evidence for the existence of another subphase $\mathrm{Sm}-C_{A}^{*}(0$ $\left.<q_{T}<1 / 3\right)$ other than Sm- $C_{A}^{*}(1 / 3)$ and Sm- $C_{A}^{*}(1 / 2)$}

We have observed textures in thick (ca. $50 \mu \mathrm{m})$ freestanding films of the corresponding mixtures used in obtaining Figs. 5(a)-5(c). Optical micrographs were taken by lowering the temperature stepwise. Some of the micrographs are summarized in Figs. 6(a)-6(e). Although we used the same oven and temperature controller, the actual sample temperatures in free-standing films were slightly different from those in homeotropic cells. The actual temperature stability is much better than $\pm 5 \mathrm{mK}\left( \pm 0.005{ }^{\circ} \mathrm{C}\right)$, but the spatial uniformity is not good as seen in Figs. 6(a)-6(e). Here we positively utilize this spatial nonuniformity together with the temporal stability. As given in Fig. 6(a), we can see a green ring produced by the Bragg reflection due to the helical structure of a possible $\mathrm{Sm}-C_{A}^{*}\left(0<q_{T}<1 / 3\right)$ subphase in nominaly pure $(S)$-12BIMF10. Contrary to the previous report [40], it is green instead of red and moreover 
(a) $\mathrm{S}-12 \mathrm{BI} \mathrm{MF} 10$

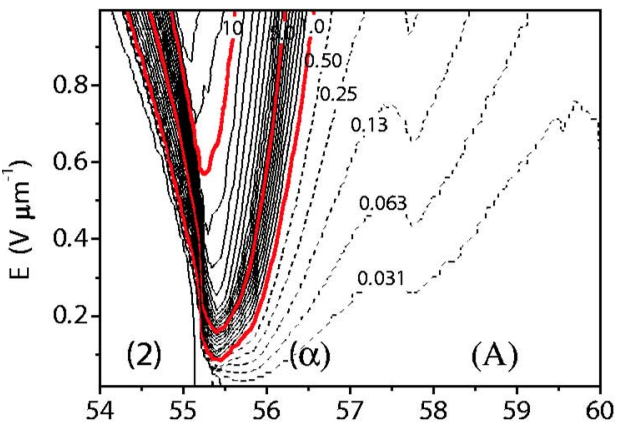

(b) (S) $-97.5 \%$

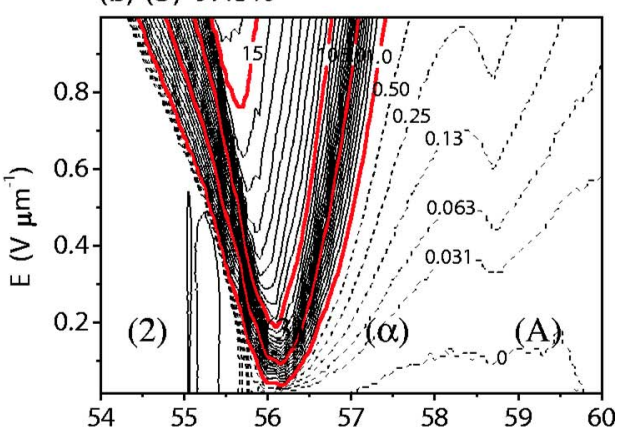

(c) $(\mathrm{S})-95 \%$

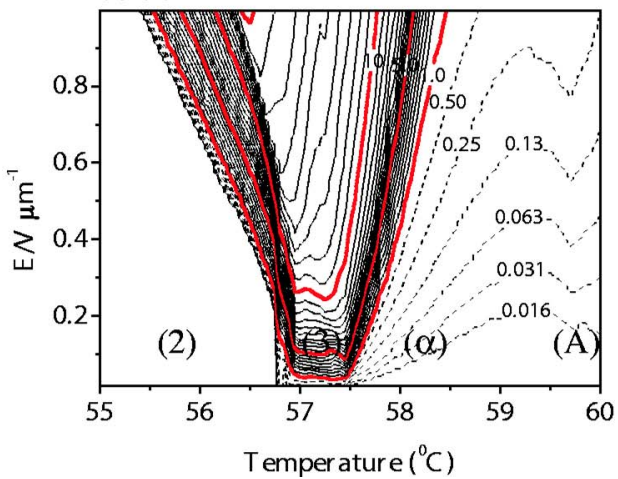

FIG. 5. (Color online) Electric-field-induced birefringence observed during the cooling cycle in the binary mixture system of $(S)$-12BIMF10 and (S)-MHPBC: (a) nominally pure $(S)$-, (b) $97.5 \%$ $(S)$-, and (c) 95\% $(S)$-12BIMF10 in wt. The birefringence contours drawn in solid lines are at steps of $0.5 \times 10^{-4}$ and some auxiliary lines in smaller steps are drawn as dotted lines. The numbers within brackets in (a) and (b) indicate the number of layers in a unit cell. $\alpha$ and $A$ denote $\mathrm{Sm}-C_{\alpha}^{*}$ and $\mathrm{Sm}-A$, respectively. The new subphase in (b) and (c) appears in a narrow temperature range in between those marked (2) and (3) (not shown due to restricted space).

Sm- $C_{A}^{*}(1 / 3)$ does not emerge. The width of the green ring corresponds to about $10 \mathrm{mK}\left(0.01{ }^{\circ} \mathrm{C}\right)$. By comparing Figs. 5(a) and 6(a), we can reasonably conclude that the hightemperature inside of the green ring is $\mathrm{Sm}-C_{\alpha}^{*}$ and the lowtemperature outside is $\mathrm{Sm}-C_{A}^{*}$. The textures are very uniform and dark on both sides, while the green ring itself shows some nonuniformity. This phase sequence, as well as the green color, indicates that the previously used $(R)$-12BIMF10 [40] was optically less pure than the $(S)$-12BIMF10 used in the present investigations.

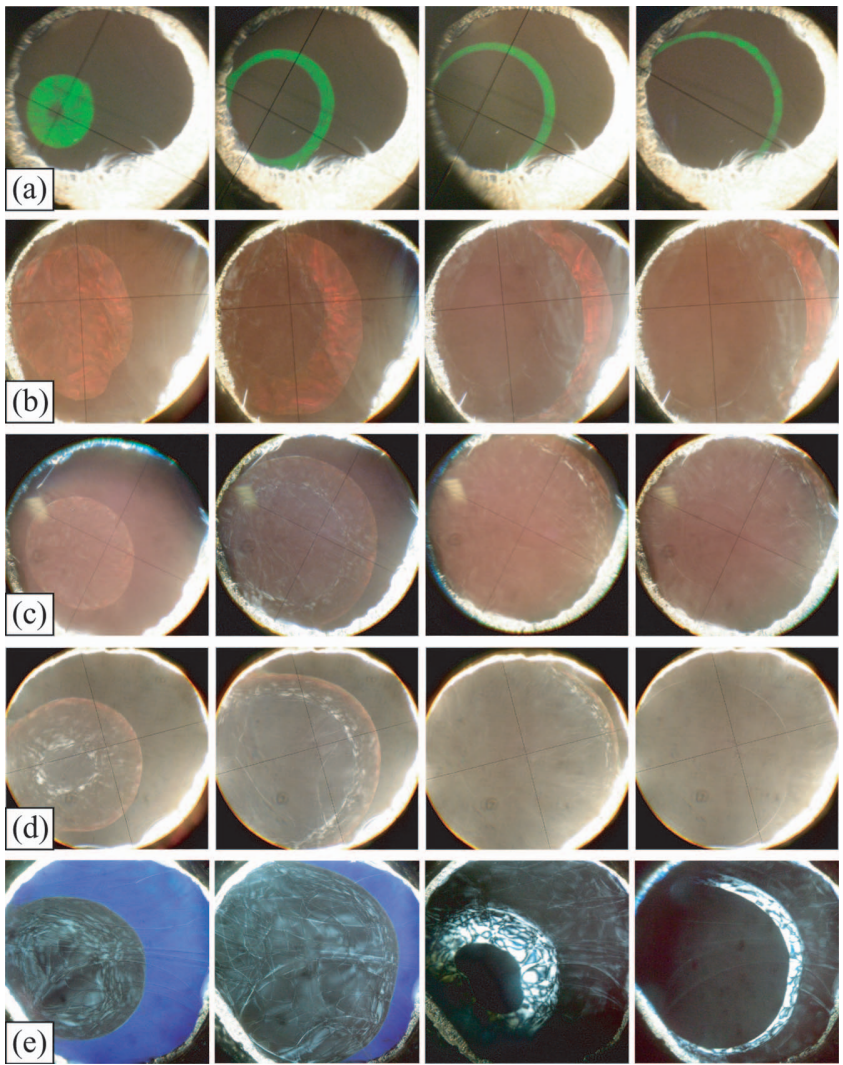

FIG. 6. (Color) Optical micrographs taken at several temperatures during the cooling cycle in free-standing films of thickness $\sim 50$, of $(S)$-12BIMF10-( $S$-MHPBC binary mixtures: (a) nominally pure $(S)_{-}$, (b) $97.5 \%(S)_{-}$, (c) $95 \%(S)$-, (d) $92 \%(S)$-, and $85 \%$ $(S)$-12BIMF10 in wt. Observed temperatures in ${ }^{\circ} \mathrm{C}$ are from left (1) to right (4): (a) 55.18, 55.20, 55.30, and 55.40; (b) 56.20, 56.50, 56.70, and 56.90; (c) 57.70, 58.30, 58.70, and 58.80; (d) 59.00, 59.30, 59.50, and 59.80; and (e) 62.00, 62.50, 63.00, and 63.40. In each photo, temperature on the right is lower than the left by approximately $0.5^{\circ} \mathrm{C}$, heater being situated on the left. In (a) the green ring is $\mathrm{Sm}-C_{A}^{*}\left(0<q_{T}<1 / 3\right)$, on the right is $\mathrm{Sm}-C_{A}^{*}$, and the region enclosed by the green ring is $\mathrm{Sm}-C_{\alpha}^{*}$. In (b) the red ring is $\mathrm{Sm}-C_{A}^{*}\left(0<q_{T}<1 / 3\right)$, on the right is $\mathrm{Sm}-C_{A}^{*}$, and on the extreme left is $\mathrm{Sm}-C_{\alpha}^{*}$, followed by $\mathrm{Sm}-C_{A}^{*}(1 / 3)$. In (c) and (d), Sm- $C_{A}^{*}(0$ $<q_{T}<1 / 3$ ) emerges from right as a pale red ring and shrinks with decreasing temperature. The right outside is $\mathrm{Sm}-C_{A}^{*}$ and the left inside is $\mathrm{Sm}-C_{A}^{*}(1 / 3)$; $\mathrm{Sm}-C_{\alpha}^{*}$ can also be seen in (c-3), (c-4), and (d-4). In (e) the violet color region is Sm- $C_{A}^{*}$ and the inside must be Sm- $C_{A}^{*}\left(0<q_{T}<1 / 3\right)$ and $\mathrm{Sm}-C_{A}^{*}(1 / 3)$ though the boundary between them is not clear. As the temperature increases, the violet color region disappears towards outside and $\mathrm{Sm}-C_{A}^{*}(1 / 2)$ emerges as a reticular ring; on the left inside is $\mathrm{Sm}-C_{\alpha}^{*}$ and the right outside is $\mathrm{Sm}-C_{A}^{*}(1 / 3)$.

Mixing (S)-12BIMF10 with $(S)$-MHPBC, which has a longer pitch, changes the Bragg-reflected color of Sm- $C_{A}^{*}\left(0<q_{T}<1 / 3\right)$ from green to red and stabilizes $\mathrm{Sm}-C_{A}^{*}(1 / 3)$ at $E=0$ in the mixture of $[12 \mathrm{BIMF} 10] /[\mathrm{MHPBC}]=97.5 / 2.5$ in weight. As shown in the (b-3) and (b-4) micrographs of Fig. 6, we can directly see the phase sequence Sm- $C_{A}^{*}-\mathrm{Sm}-C_{A}^{*}\left(0<q_{T}<1 / 3\right)-\mathrm{Sm}-C_{A}^{*}(1 /$ $3)-\mathrm{Sm}-C_{\alpha}^{*}$ from the low-temperature outside to the hightemperature inside of the red ring. The quite uniform texture 


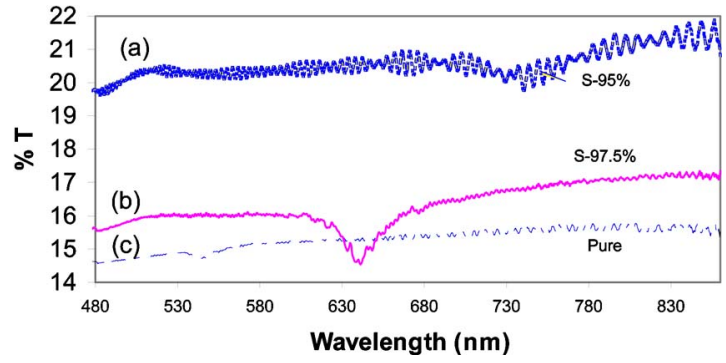

FIG. 7. (Color online) Transmittance spectra measured at oblique incidence of $20^{\circ}$ in free-standing films of $(S)$-12BIMF10$(S)$-MHPBC binary mixtures: (a) nominally pure (S)-, (b) $97.5 \%$ (S)-, and (c) $95 \%$ (S)-12BIMF10 in wt.

observed around the highest-temperature central part of the micrographs represents $\mathrm{Sm}-C_{\alpha}^{*}$, and the slightly rugged texture between $\mathrm{Sm}-C_{\alpha}^{*}$ and the red ring $\left[\mathrm{Sm}-C_{A}^{*}\left(0<q_{T}\right.\right.$ $<1 / 3)]$ is $\mathrm{Sm}-C_{A}^{*}(1 / 3)$. The optical transmittance spectra of the films of nominally pure, $97.5 \%$ and $95 \%$, (S)-12BIMF10 recorded at oblique incidence angle of $20^{\circ}$ as shown in Fig. 7 exhibit small dips corresponding to Bragg reflection at wavelengths of 541,642 , and $740 \mathrm{~nm}$ at 55.20, 56.70, and $58.7^{\circ} \mathrm{C}$, respectively. The full-pitch band was not observed in the transmittance spectrum at oblique incidence for nominally pure, $97.5 \%$ and $95 \%$, (S)-12BIMF10 at the measured temperatures. To confirm this phase sequence and to study the phase properties more quantitatively, we tried to obtain the ORP and $E-T$ phase diagram mapped with birefringence contours in both the cooling and heating cycles by using a $25-\mu \mathrm{m}$-thick homeotropic cell. Before applying any field, we first measured the ORP and the residual birefringence in the cooling or heating cycles with a rate of $10 \mathrm{mK} / \mathrm{min}$ $\left(0.01{ }^{\circ} \mathrm{C} / \mathrm{min}\right)$. The time constant of the lock-in amplifier was set at $1 \mathrm{~s}$. Then we measured the field-induced birefringence at a particular temperature by changing the applied field in 100 steps. The temperature was lowered or raised stepwise at a rate of $0.01{ }^{\circ} \mathrm{C} / \mathrm{min}$ or less. To assure the thermal equilibrium, the sample was kept at each measuring temperature for $1 \mathrm{~min}$ before starting to change the field. The results are summarized in Fig. 8. The field-induced birefringence measurements deteriorate the cell quality and shift the residual birefringence level. This happens mainly in Sm- $C_{A}^{*}$ in the cooling cycle and mainly in $\mathrm{Sm}-C_{\alpha}^{*}$ in the heating cycle.

The macroscopic helical pitch in $\mathrm{Sm}-C_{A}^{*}$ is so short that the Bragg reflection is not observed in the visible region. This results in the considerably uniform texture in Fig. 6 and nearly zero ORP signal in Fig. 8. Although the residual birefringence in $\mathrm{Sm}-C_{A}^{*}$ is not zero in the cooling cycle, it is much less than that observed in $\operatorname{Sm}-C_{A}^{*}\left(0<q_{T}<1 / 3\right)$ and $\mathrm{Sm}-C_{A}^{*}(1 / 3), 0.3 \times 10^{-4}$. Because of its short-pitch helical structure, $\mathrm{Sm}-C_{\alpha}^{*}$ is optically uniaxial and hence the texture is quite uniform as seen in Fig. 6; the ORP and residual birefringence are zero in the cooling and heating cycles. Aside from some minor differences, Figs. 8(a) and 8(b) assure the existence of $\mathrm{Sm}-C_{A}^{*}\left(0<q_{T}<1 / 3\right)$ as well as $\mathrm{Sm}-C_{A}^{*}(1 / 3)$ at $E=0$. The Bragg reflection peak of the helical structure of Sm- $C_{A}^{*}\left(0<q_{T}<1 / 3\right)$ almost coincides with the wavelength of the $\mathrm{He}-\mathrm{Ne}$ laser $(633 \mathrm{~nm})$. Since the helical structure can be modified easily by the interface effect in free-standing films, the Bragg reflection peak may show some slight shift. Actually, the cooling and heating processes make it longer and shorter than $633 \mathrm{~nm}$, respectively; the (a)

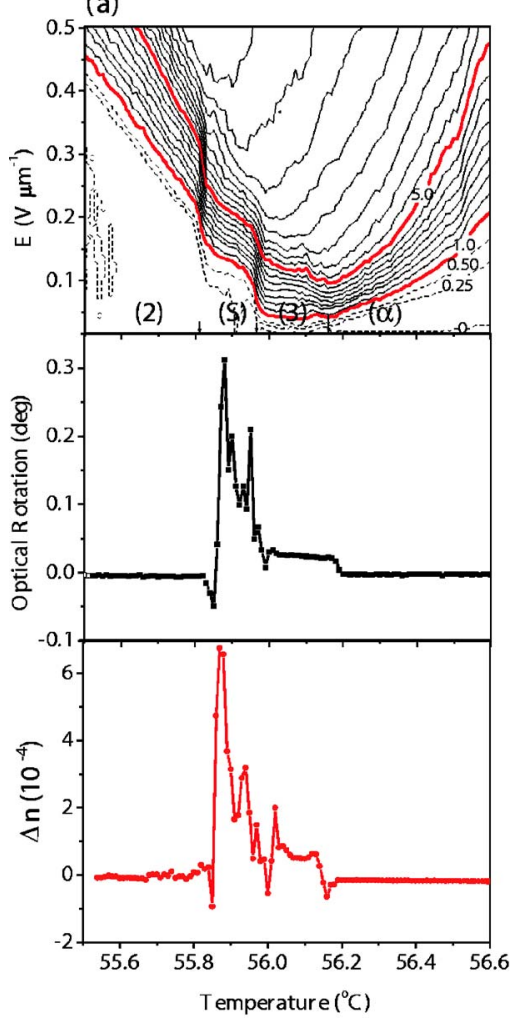

(b)

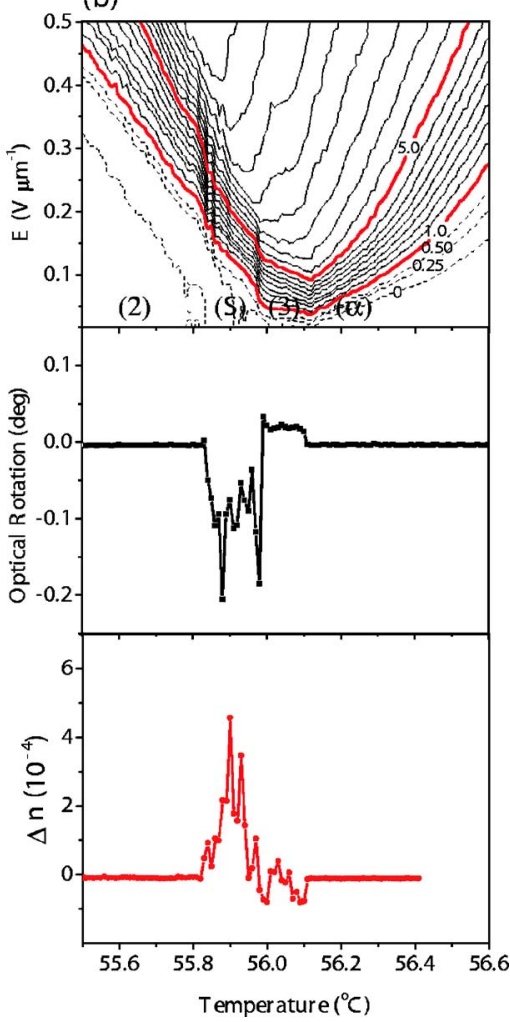

FIG. 8. (Color online) Electric-field-induced birefringence, optical rotation $(\mathrm{ORP}=$ optical rotation/sample thickness), and residual birefringence measured in (a) cooling and (b) heating cycles in $25-\mu \mathrm{m}$ homeotropic cells of $97.5 \mathrm{wt} \%$ 12BIMF10. This clearly shows the existence of an additional subphase $\mathrm{Sm}-C_{A}^{*}\left(0<q_{T}<1 / 3\right)$ in the cooling and heating processes. The birefringence contours drawn in solid lines are at steps of $0.5 \times 10^{-4}$ and some auxiliary lines in smaller steps are drawn in dotted lines. The numbers within brackets in (a) and (b) indicate the number of layers in a unit cell. $\alpha$ and $S$ are $\mathrm{Sm}-C_{\alpha}^{*}$ and the subphase, respectively. 


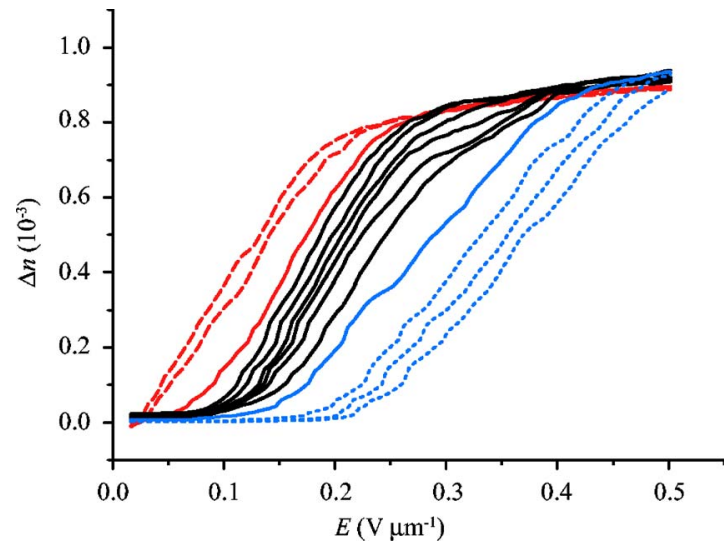

FIG. 9. (Color online) Original data of $\Delta n$ vs $E$ obtained by changing the field at steps of $1 \mathrm{mV} / \mu \mathrm{m}$ and the temperature at $20 \mathrm{mK}\left(0.02{ }^{\circ} \mathrm{C}\right)$ steps in $97.5 \mathrm{wt} \% 12 \mathrm{BIMF} 10$ for the temperature range of $55.76-56.00{ }^{\circ} \mathrm{C}$. Solid lines are for $\mathrm{Sm}-C_{A}^{*}\left(0<q_{T}\right.$ $<1 / 3)$, and dotted and dashed lines are for $\operatorname{Sm}-C_{A}^{*}(0)$ and $\mathrm{Sm}-C_{A}^{*}(1 / 3)$ (i.e., two and three layers), respectively. The increase of temperature shifts the curves to the left of the figure. Note that there clearly exists threshold in the new subphase.

ORP is observed to be positive and negative as shown in Figs. 8(a) and 8(b) accordingly. This interface effect may also be responsible for the very noisy ORP signal corresponding to the red band; when the wavelength used for the ORP measurement almost coincides with the Bragg reflection peak, the signal intensity critically depends on the peak wavelength that may easily be changed somewhat by the interface effect.

In this way, we have firmly established that there exists an additional subphase, $\mathrm{Sm}-C_{A}^{*}\left(0<q_{T}<1 / 3\right)$, between $\mathrm{Sm}-C_{A}^{*}$ and $\mathrm{Sm}-C_{A}^{*}(1 / 3)$ in the mixture of [12BIMF10]/[MHPBC] $=97.5 / 2.5$. It is located just below $\mathrm{Sm}-C_{A}^{*}(1 / 3)$ and looks like a part of $\mathrm{Sm}-C_{A}^{*}$ in the $E-T$ phase diagram. This may indicate that the additional subphase is antiferroelectric. In order to see whether it has a net spontaneous polarization and hence immediately responds to an applied field, we checked the original data used for obtaining the $E-T$ phase diagram mapped with birefringence contours shown in Fig. 8(a). As described above, we measured birefringence by scanning the field at various temperatures. Figure 9 illustrates some of the data obtained at every steps of $20 \mathrm{mK}$ from 56.00 to $55.76{ }^{\circ} \mathrm{C}$. Two dashed (red) lines represent the response at temperatures of 56.00 and $55.98^{\circ} \mathrm{C}$ in $\mathrm{Sm}-C_{A}^{*}(1 / 3)$; the birefringence is linearly dependent on the applied electric field near $E=0$, since $\mathrm{Sm}-C_{A}^{*}(1 / 3)$ is ferrielectric and has net spontaneous polarization. On the other hand, three dotted (blue) lines show the field-induced birefringence at $55.80,55.78$, and $55.76^{\circ} \mathrm{C}$ in $\mathrm{Sm}-C_{A}^{*}$. The birefringence does not immediately increase but stays at zero near $E=0$. At much lower temperatures, the birefringence first becomes negative because the in-layer directors first tend to become parallel to the applied field during the unwinding process of the macroscopic helical structure as already pointed out concerning Fig. 4. Clearly the threshold exists, since $\mathrm{Sm}-C_{A}^{*}$ is antiferroelectric. Between temperatures of 55.96 and $55.82{ }^{\circ} \mathrm{C}$, all of the six solid (black) lines belong unambiguously to $\mathrm{Sm}-C_{A}^{*}\left(0<q_{T}<1 / 3\right)$ and show the zero birefringence range near $E=0$ although the threshold field is lower than that of $\mathrm{Sm}-C_{A}^{*}$. Note that the marginal two solid (red and blue) lines seem to be affected by the finite spot size of the laser beam light and the spatial temperature nonuniformity in the oven used in the field-induced birefringence measurements. The existence of the zero-birefringence range near $E=0$ indicates that $\mathrm{Sm}-C_{A}^{*}\left(0<q_{T}<1 / 3\right)$ is an antiferroelectric subphase.

According to the Emelyanenko-Osipov model recently reconsidered in greater detail by Emelyanenko, Fukuda, and Vij [43], the subphase with the simplest structure (the smallest unit cell) below the three-layer phase is $\mathrm{Sm}-C_{A}^{*}(1 / 5)$. However, the observed subphase is antiferroelectric in nature since it is found to be having a finite threshold and the fullpitch band at oblique incidence is absent. This subphase is also different from the two- and four-layer structures; the next logical identification of this subphase could therefore be an eight-layer structure with $q_{T}=1 / 4$. Thus we tentatively assign the red band observed in the mixture of [12BIMF10]/[MHPBC]=97.5/2.5 as Sm- $C_{A}^{*}(1 / 4)$. The corresponding bands with the green and near-IR Bragg reflection were also studied in detail in nominally pure $(S)$-12BIMF10 and another mixture of $[12 \mathrm{BIMF} 10] /[\mathrm{MHPBC}]=95 / 5$ as shown in Fig. 10. The threshold is confirmed to exist as given in Fig. 11. It is worthwhile to note that $\mathrm{Sm}-C_{A}^{*}(1 / 4)$ emerges stably at $E=0$ while $\mathrm{Sm}-C_{A}^{*}(1 / 3)$ is not stable in the nominaly pure (S)-12BIMF10.

\section{DISCUSSION}

Two extreme models that had been proposed to explain the emergence of the optically uniaxial and biaxial subphases were the Ising model $[15-19,44,45]$ and the clock $(X-Y)$ model [46-50]. Since the tilted smectic liquid crystals have the azimuthal angle freedom of the director tilting direction, the clock model appears to be suitable for the understanding of the formation of large-scale subphase structures with modulation periods of several smectic layers. However, the azimuthal angle modulation might occur continuously and it was not easy to explain the staircase emergence of the biaxial subphases. In fact, Roy and Madhusudana included the longrange interactions between the third- (and even the fourth-) nearest layers and the reference layer in order to successfully obtain $\mathrm{Sm}-C_{A}^{*}(1 / 2)$ with four-layer periodicity, and an electric field induced $\mathrm{Sm}-C_{A}^{*}(1 / 3)$ with the three-layer periodicity. Without these long-range interactions, they could not really stabilize these subphases $[48,49]$. How, in practice, can we really envisage such long-range interactions between the fourth-nearest layers and the reference layer in such a disorderd system of liquid crystals to occur?

On the other hand, the Ising model has been used for the understanding of the formation of large-scale structures frequently encountered in solid-state physics-for instance, in binary alloys, near hcp-fcc structural transitions, in intercalated graphite, and in many other systems [51]. What is common in these cases is the presence of some form of frustra- 
(a)

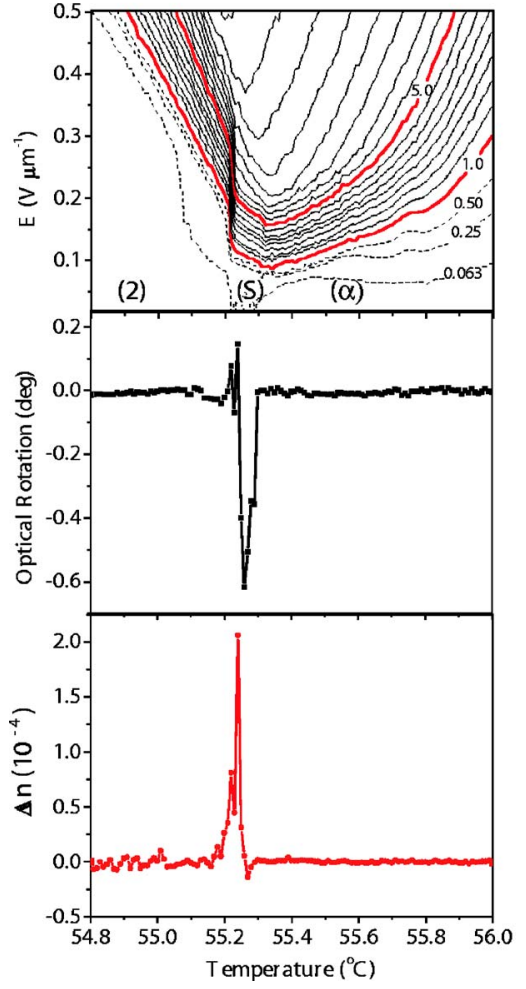

(b)

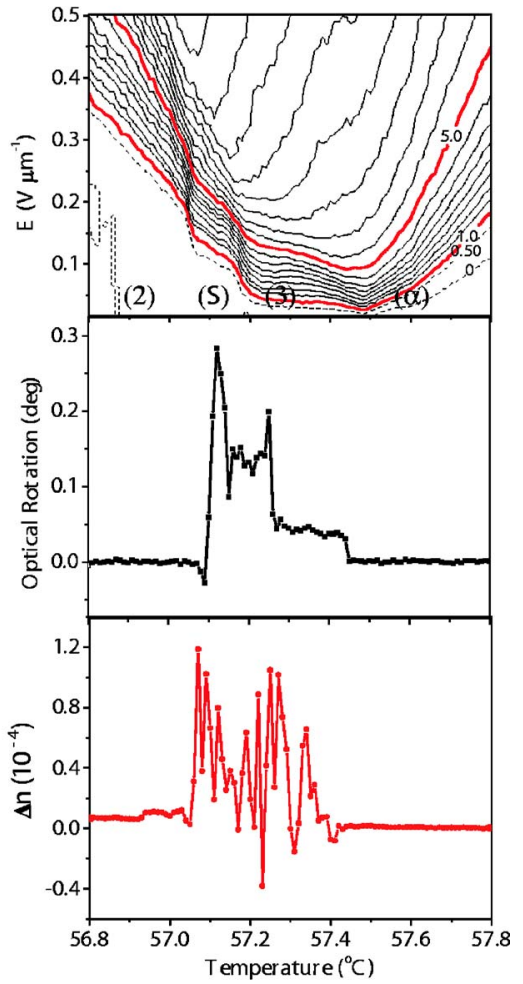

FIG. 10. (Color online) Electric-field-induced birefringence, optical rotation $(\mathrm{ORP}=$ optical rotation/sample thickness), and residual birefringence measured in the cooling cycle in $25-\mu \mathrm{m}$ homeotropic cells of (a) nominally pure and (b) 95.0 wt \% 12BIMF10. This clearly shows the existence of an additional subphase $\mathrm{Sm}-C_{A}^{*}\left(0<q_{T}\right.$ $<1 / 3)$ in the cooling process in both nominally pure and $95 \mathrm{wt} \%$ 12BIMF10. The birefringence contours drawn in solid lines are at steps of 0.5 $\times 10^{-4}$ and some auxiliary lines in smaller steps are drawn in dotted lines. The numbers within brackets in (a) and (b) indicate the number of layers in a unit cell. $\alpha$ and $S$ are $\mathrm{Sm}-C_{\alpha}^{*}$ and the subphase, respectively. tion in the phase diagram. At the point where the dominant ordering force of a system happens to change sign, a large number of alternative structures may acquire exactly the same free energy. This degeneracy can be lifted by weak long-range forces or thermal fluctuations. Statistical mechanics models have been developed which exemplify these two different ways of lifting the degeneracy: the one-dimensional Ising model with the long-range repulsion $[52,53]$ and the so-called ANNNI (axial next-nearest-neighbor Ising) model with competing nearest- and next-nearest-neighbor couplings $[54,55]$. In the tilted smectic liquid crystals under consideration, the system is frustrated between the synclinic ferroelectric $\mathrm{Sm}-C^{*}$ and anticlinic antiferroelectric $\mathrm{Sm}-C_{A}^{*}$. Moreover, almost all intermolecular interactions tend to force the

(a)

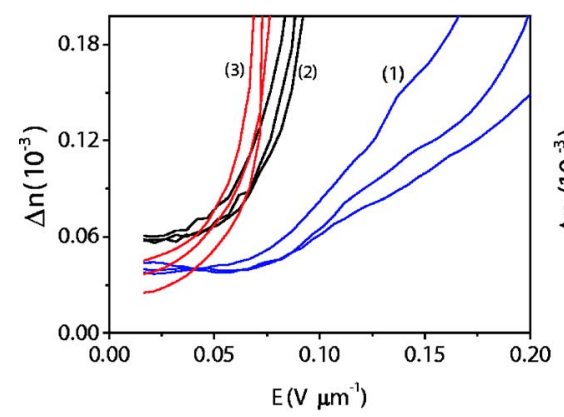

molecules to align parallel to a plane; it is natural and simpler to use the Ising model for understanding the emergence of such large-scale subphase structures. Yamashita and coworkers applied the ANNNI model and published a series of papers $[17,44]$. Since the molecular orientational order parameter in the present case is as large as 0.7 or higher, however, it is hardly considered that thermal fluctuations alone can lift the degeneracy and produce the large-scale subphase structures; the ANNNI model Hamiltonian cannot be applied in dealing with the frustration between synclinic ferroelectric Sm- $C^{*}$ and anticlinic antiferroelectric $\mathrm{Sm}-C_{A}^{*}$.

In the early stages of investigations, Isozaki et al. $[15,16]$ applied the one-dimensional Ising model with long-range repulsion and introduced the $q_{T}$ number, as already pointed out

(b)

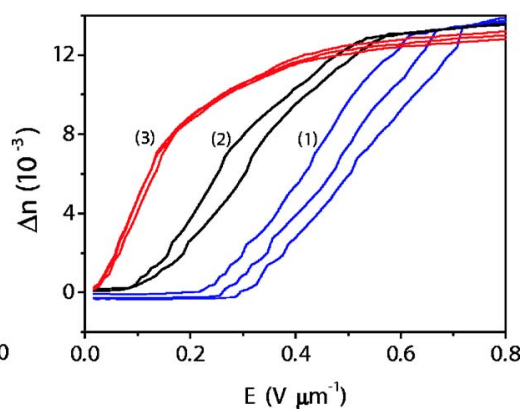

FIG. 11. (Color online) Original data of $\Delta n$ vs $E$ obtained by changing the field at $1 \mathrm{mV} / \mu \mathrm{m}$ steps and the temperature at $20 \mathrm{mK}\left(0.02{ }^{\circ} \mathrm{C}\right)$ steps, (a) in nominally pure $(S)$-12BIMF10 for the temperature range of $55.15-55.35{ }^{\circ} \mathrm{C}$ (but for relatively low electric fields), and (b) $95.0 \mathrm{wt} \%$ for the temperature range of $57.04-57.24{ }^{\circ} \mathrm{C}$. Note that the measurements are made under the sample being cooled. In (a) and (b) black solid lines, (2), are for $\operatorname{Sm}-C_{A}^{*}\left(0<q_{T}<1 / 3\right)$, red solid lines, (3), for adjacent high temperature $\mathrm{Sm}-C_{\alpha}^{*} / \mathrm{Sm}-C_{A}^{*}(1 / 3)$ phase and blue solid lines, (1), for adjacent low temperature $\mathrm{Sm}-C_{A}^{*}$ phase. The increase of temperature shifts the curves to the left of the figure. Note that there clearly exists threshold in this subphase. 


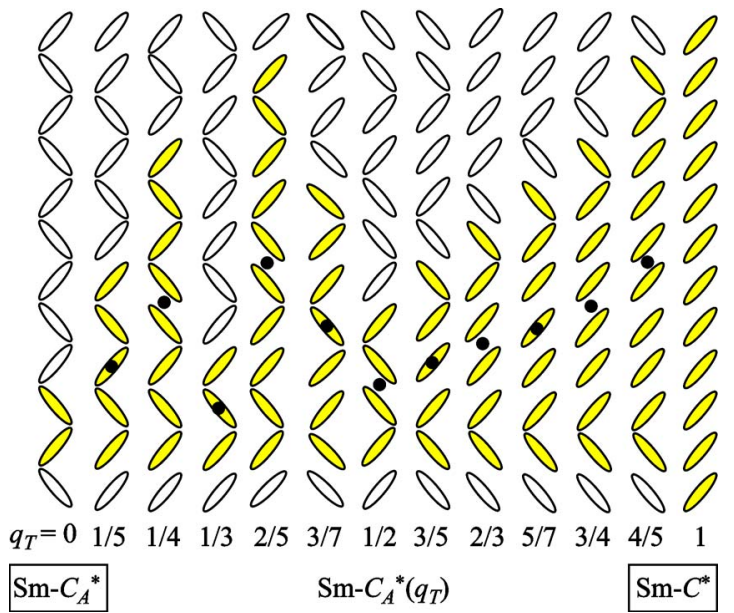

FIG. 12. (Color online) Possible subphase sequence. Biaxial subphases between $\mathrm{Sm}-C_{A}^{*}$ and $\mathrm{Sm}-C^{*}$ are designated as $\operatorname{Sm}-C_{A}^{*}\left(q_{T}\right)$ with ferroelectric, $[F]$, and antiferroelectric orderings, $[A]$, and characterized by a fraction of the ferroelectric ordering within a unit cell by $q_{T}=[F] /([A]+[F])$. The tilt directions in different layers are antisymmetric with respect to the middle (the black dot) of the shaded (yellow colored) molecules. In other words, the biaxial subphase $\mathrm{Sm}-C_{A}^{*}\left(q_{T}\right)$ has a microscopic (short-pitch) helical structure, though highly distorted, and the pitch is given by $2 /(1$ $\left.-q_{T}\right)$ in the unit of the number of smectic layers. At the same time, it has a macroscopic ordinary helical structure with the long pitch given by Eq. (12).

in Sec. I. The free energy of a subphase specified by $q_{T}$ is given by

$$
F_{q_{T}}=\left\{\left(1-q_{T}\right) F_{C A}+q_{T} F_{C}\right\}+\text { (long-range interactions), }
$$

where $F_{C A}$ and $F_{C}$ are the free energies for $\mathrm{Sm}-C_{A}^{*}$ and $\mathrm{Sm}-C^{*}$, respectively. Without taking account of the longrange interactions, the only stable phases are $\mathrm{Sm}-C_{A}^{*}$ and $\mathrm{Sm}-C^{*}$ and the degeneracy at the frustration point-i.e., the phase transition temperature between them is not lifted. They first assumed repulsive forces between the $F$ orderings; any subphase structure expected to appear is specified by an irreducible rational number $q_{T}$, which constitutes a Farey sequence given in Fig. 12.

The two prototype subphases were appropriately identified as ferrielectric $\mathrm{Sm}-C_{A}^{*}(1 / 3)$ and antiferroelectric $\mathrm{Sm}-C_{A}^{*}(1 / 2)$. The macroscopic helical pitch of $\mathrm{Sm}-C_{A}^{*}\left(q_{T}\right)$ was considered to be given by

$$
\frac{1}{p\left(\operatorname{Sm} C_{A}^{*}\left(q_{T}\right)\right)}=-\frac{q_{T}}{p\left(\operatorname{Sm} C^{*}\right)}+\frac{1-q_{T}}{p\left(\operatorname{Sm} C_{A}^{*}\right)} .
$$

This equation is based on the empirically established fact that the macroscopic helical pitch of Sm- $C_{A}^{*}$ consisting of the $A$ orderings only and of Sm- $C^{*}$ consisting of the $F$ orderings only are nearly equal to each other but with their handedness being just opposite to each other. Consequently, the helical pitch in $\mathrm{Sm}-C_{A}^{*}(1 / 3)$ and $\mathrm{Sm}-C_{A}^{*}(1 / 2)$ must be very long; it may become infinite either in $\mathrm{Sm}-C_{A}^{*}(1 / 3)$ as actually seen in Fig. 4 or in $\operatorname{Sm}-C_{A}^{*}(1 / 2)$ as given in [56]. Furthermore, such

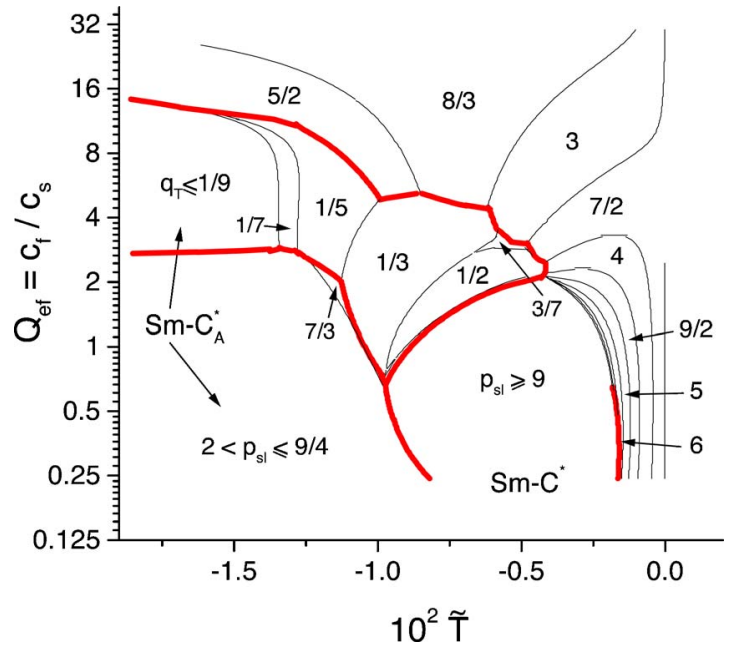

FIG. 13. (Color online) Effective quadrupole-reduced temperature phase diagram in the case of constant $\tilde{c}_{s} \tilde{c}_{f}$. Zero temperature corresponds to the transition into Sm- $A$. In between the two thick (red) lines, biaxial subphases are indicated with $q_{T}$ values. On the right-hand side of the thick (red) line, the uniaxial subphases are indicated by $p_{s l}$ (the number of smectic layers in one period) The thick (red) line in the vicinity of $\widetilde{T}=0$ corresponds to the first order phase transition between Sm- $C^{*}$ and $\mathrm{Sm}-C_{\alpha}^{*}$. Above this line a continuous (although very sharp) modification of $\mathrm{Sm}-C^{*}$ into $\mathrm{Sm}-C_{\alpha}^{*}$ is observed.

simple Ising model can explain the intrinsic instability observed in $\mathrm{Sm}-C_{A}^{*}(1 / 3)$, where there exists a net spontaneous polarization and the helical pitch is long. Since the free energy difference between the $A$ and $F$ orderings is small, solitonlike defects in the orderings produced by misplacing the $A$ and $F$ orderings must be thermally excited spatially at random to neutralize the spontaneous polarization locally, causing fluctuations in $\mathrm{Sm}-C_{A}^{*}(1 / 3)$. The fluctuations explain the low-frequency dielectric responses observed by Glogarova $e t$ al. [57] and the very slow relaxation time observed by Miyachi et al. [58] in dynamic Rayleigh light scattering.

In this way the Ising model has been quite successful in explaining several aspects of experimental observations; however, it could neither clarify, from its nature, the nonplanar structures of the biaxial subphases [24-36] nor the emergence of uniaxial $\mathrm{Sm}-C_{\alpha}^{*}$ characterized by the short-pitch helical structure [59-68]. An introduction of the novel Casimirtype long-range forces did not eliminate the above difficulties in the Ising model $[18,19,45]$. The EmelyanemkoOsipov model removes all of these difficulties in a clear and straightforward manner by introducing the discrete flexoelectric polarization as detailed in Sec. I. According to the theoretical model presented in $[20,21,43]$, the new observed phase may be either $\mathrm{Sm}-C_{A}^{*}(1 / 5)$ or $\mathrm{Sm}-C_{A}^{*}(1 / 4)$. In this model the molecular transverse dipole moment associated with the polar bonds near the chiral center and the central quadrupole moment associated with the aromatic rings appear to play the most important role. The theoretical quadrupole-temperature phase diagram is presented in Fig. 13, where the thick (red) lines detach the biaxial phases from the uniaxial ones and $\mathrm{Sm}-\mathrm{C}^{*}$ from $\mathrm{Sm}-C_{\alpha}^{*}$ in the case of an abrupt transition between them. The uniaxial phases are 
marked by $p_{s l}$ denoting the number of smectic layers per single pitch, and the biaxial phases are marked by $q_{T}$ denoting the ratio of synclinic pairs to the sum of synclinic and anticlinic pairs per one unit cell. The well-known Sm $-C_{A}^{*}(1 / 3)$ and $\mathrm{Sm}-C_{A}^{*}(1 / 2)$ appear to be the most stable phases in the dominant range of the temperature and quadrupole moment, though different biaxial subphases with $q_{T}$ $<1 / 3$ and even with $1 / 3<q_{T}<1 / 2$ and $q_{T}>1 / 2$ become more stable within some temperature ranges. Biaxial subphases with $q_{T} \ll 1$ and uniaxial subphases with $p_{s l} \sim 2$ look already very similar to each other and to $\mathrm{Sm}-C_{A}^{*}(0)$ and exist within the small temperature intervals, and thus, observable temperature range of these phases is small. The temperature range in which the biaxial subphases $1 / 3<q_{T}<1 / 2$ and $q_{T}$ $>1 / 2$ can be observed is also miserably restricted. However, one notes from Fig. 13 that, for example, $\mathrm{Sm}-C_{A}^{*}(1 / 5)$ may arise over a quite larger temperature range, and it is essentially different from $\mathrm{Sm}-C_{A}^{*}(0), \quad \mathrm{Sm}-C_{A}^{*}(1 / 3)$, and $\mathrm{Sm}-C_{A}^{*}(1 / 2)$ and thus, it may, in principle, correspond to the new observed subphase.

The phase sequence for pure $(S)$-MHPBC is $\mathrm{Sm}-C_{A}^{*}(0)$, $\mathrm{Sm}-C_{A}^{*}(1 / 3), \quad \mathrm{Sm}-C_{A}^{*}(1 / 2)$, and $\mathrm{Sm}-C_{\alpha}^{*}$ [see Fig. 4(a)] and, thus, the effective quadrupole moment $Q_{e f}$ (that is proportional to the ratio of the flexoelectric constant $c_{f}$ depending on the real quadrupole moment and parameter $c_{s}$ representing molecular chirality) for this material seems to be $\sim 2$ (see Fig. 13). Partial racemization of $(S)$-MHPBC leads to the appearance of Sm- $C^{*}$ between $\mathrm{Sm}-C_{A}^{*}(1 / 2)$ and $\mathrm{Sm}-C_{\alpha}^{*}$, and simultaneously to the decrease of the temperature range for $\mathrm{Sm}-C_{A}^{*}(1 / 2)$ [see Fig. 4(b)]. In Fig. 13 this sequence is also possible for smaller $c_{f} / c_{s}$. One notes, however, that racemization must enlarge the ratio $c_{f} / c_{s}$. This discrepancy arises because Fig. 13 is plotted for the constant product $c_{s} c_{f}$ of these coefficients, whereas racemization diminishes this product, and thus, Fig. 13 itself cannot reproduce the racemization process in a proper way. If $c_{s} c_{f}$ is rescaled, a new diagram will reproduce the desired sequence for the proper ratio of $c_{f} / c_{s}$.

The phase sequence for pure 12BIMF10 [see Fig. 10(a)] is $\mathrm{Sm}-C_{A}^{*}(0), \mathrm{Sm}-C_{A}^{*}\left(q_{T}<1 / 3\right)$, and $\mathrm{Sm}-C_{\alpha^{*}}^{*}$. One notes that this sequence is well demonstrated in theoretical phase diagram (Fig. 13) beginning from $Q_{e f} \sim 6$, and a sequence $\mathrm{Sm}-C_{A}^{*}(0), \mathrm{Sm}-C_{A}^{*}\left(q_{T}<1 / 3\right), \mathrm{Sm}-C_{A}^{*}(1 / 3)$, and Sm- $C_{\alpha}^{*}$ typical for the mixture of 12BIMF10 and MHPBC [see Fig. 10(b)] is properly reproduced in Fig. 13 at smaller $Q_{e f}$.

\section{CONCLUSIONS}

We have confirmed the existence of the biaxial subphase other than the three- and four-layer phases between $\mathrm{Sm}-C_{A}^{*}(0)$ and $\mathrm{Sm}-C_{A}^{*}(1 / 3)$ in the antiferroelectric liquidcrystalline compound of $(S)$-12BIMF10 by studying the Bragg reflections, the electric-field-induced birefringence, and the optical rotatory power. This temperature range of the subphase region in $(S)$-12BIMF10 becomes wider and the length of the pitch increases when it is mixed with $(S)$-MHPBC, which has a longer pitch and opposite sense of handedness. Furthermore, in this subphase region a full-pitch Bragg reflection band has not been observed in the transmittance spectra at oblique incidence for nominally pure $(S)$-12BIMF10 and binary mixtures of $(S)$-12BIMF10 and $(S)$-MHPBC. The subphase exhibits clearly a finite threshold field, though lower than that in $\mathrm{Sm}-C_{A}^{*}(0)$, suggesting the antiferroelectric structure which is consistent with the absence of the full-pitch band at oblique incidence.

The behavior of this subphase is entirely different from that of the three- and four-layer subphases and the only possible antiferroelectric structure, which can possibly exist below the three-layer is the eight-layer antiferroelectric structure in a unit cell $\left(q_{T}=1 / 4\right)$. Therefore we tentatively assign this subphase to $\mathrm{Sm}-C_{A}^{*}(1 / 4)$. According to the theory presented here, the five-layer structure $\left(q_{T}=1 / 5\right)$ rather than the eight-layer $\left(q_{T}=1 / 4\right)$ is more stable. This is presumably due to the fact that the present theoretical model considers the maximum number of nine layers due to computational reasons. Whether the theory can be extended to produce a stable eight-layer or a five-layer structure can have a finite threshold and no full pitch-band remains an open question to be resolved in the future.

\section{ACKNOWLEDGMENTS}

We thank Kashima Oil Co. ltd and Showa Shell Sekiyu K. $\mathrm{K}$. for supplying the liquid-crystal compounds 12BIMF10 and MHPBC, respectively, and the Science Foundation of Ireland (02/IN.1/I031) for funding the research work in Dublin. A.F. was funded by Trinity College Dublin under SFI Grant No. 02/W/I02. A.D.L.C. is funded by a PRTLI grant from HEA of Ireland whom we acknowledge. A.V.E. acknowledges the support of Russian Ministry of Science Grant No. MK 4007-2004 and RFBR Grant No. 04-0332096. N.M.S. is indebted to RFBR Grant No. 03-02-17288 for partial support.
[1] R. B. Meyer, L. Liebert, L. Strzelecki, and P. Keller, J. Phys. (Paris) 36, L69 (1975).

[2] R. B. Meyer, Mol. Cryst. Liq. Cryst. 40, 33 (1977).

[3] S. A. Pikin and V. L. Indenbom, Sov. Phys. Usp. 21, 487 (1978).

[4] A. D. L. Chandani, Y. Ouchi, H. Takezoe, A. Fukuda, K. Terashima, K. Furukawa, and A. Kishi, Jpn. J. Appl. Phys., Part 2 28, L1261 (1989).
[5] A. D. L. Chandani, E. Gorecka, Y. Ouchi, H. Takezoe, and A. Fukuda, Jpn. J. Appl. Phys., Part 2 28, L1265 (1989).

[6] H. R. Brand, P. E. Cladis, and H. Pleiner, Macromolecules 25, 7223 (1992).

[7] P. E. Cladis and H. R. Brand, Liq. Cryst. 14, 1327 (1993).

[8] M. A. Osipov and A. Fukuda, Phys. Rev. E 62, 3724 (2000).

[9] Y. Takanishi, A. Ikeda, H. Takezoe, and A. Fukuda, Phys. Rev. E 51, 400 (1995). 
[10] H. Sun, H. Orihara, and Y. Ishibashi, J. Phys. Soc. Jpn. 62, 2706 (1993).

[11] M. Fukui, H. Orihara, Y. Yamada, N. Yamamoto, and Y. Ishibashi, Jpn. J. Appl. Phys., Part 2 28, L849 (1989).

[12] E. Gorecka, A. D. L. Chandani, Y. Ouchi, H. Takezoe, and A. Fukuda, Jpn. J. Appl. Phys., Part 1 29, 131 (1990).

[13] H. Takezoe, J. Lee, Y. Ouchi, and A. Fukuda, Mol. Cryst. Liq. Cryst. 202, 85 (1991).

[14] N. Okabe, Y. Suzuki, I. Kawamura, T. Isozaki, H. Takezoe, and A. Fukuda, Jpn. J. Appl. Phys., Part 2 31, L793 (1992).

[15] T. Isozaki, T. Fujikawa, H. Takezoe, A. Fukuda, T. Hagiwara, Y. Suzuki, and I. Kawamura, Jpn. J. Appl. Phys., Part 2 31, L1435 (1992).

[16] A. Fukuda, Y. Takanishi, T. Isozaki, K. Ishikawa, and H. Takezoe, J. Mater. Chem. 4, 997 (1994) and references threin.

[17] T. Matsumoto, A. Fukuda, M. Johno, Y. Motoyama, T. Yui, S.-S. Seomun, and M. Yamashita, J. Mater. Chem. 9, 2051 (1999) and references therein.

[18] M. A. Osipov, A. Fukuda, and H. Hakoi, in Proceedings of Anglo-Japanese Joint Workshop held at Southampton, 2000 [Mol. Cryst. Liq. Cryst. Sci. Technol., Sect. A 402, 9 (2003)].

[19] A. Fukuda, H. Hakoi, M. Sato, and M. A. Osipov, Mol. Cryst. Liq. Cryst. 398, 169 (2003).

[20] A. V. Emelyanenko and M. A. Osipov, Phys. Rev. E 68, 051703 (2003).

[21] N. M. Shtykov, A. D. L. Chandani, A. V. Emelyanenko, A. Fukuda, and J. K. Vij, Phys. Rev. E 71, 021711 (2005).

[22] M. Cepic, E. Gorecka, D. Pociecha, B. Zeks, and H. T. Nguyen, J. Chem. Phys. 117, 1817 (2002).

[23] M. Cepic and B. Zeks, Phys. Rev. Lett. 87, 085501 (2001).

[24] P. Mach, R. Pindak, A.-M. Levelut, P. Barois, H. T. Nguyen, C. C. Huang, and L. Furenlid, Phys. Rev. Lett. 81, 1015 (1998).

[25] P. Mach, R. Pindak, A.-M. Levelut, P. Barois, H. T. Nguyen, H. Baltes, M. Hird, K. Toyne, A. Seed, J. W. Goodby, C. C. Huang, and L. Furenlid, Phys. Rev. E 60, 6793 (1999).

[26] A.-M. Levelut and B. Pansu, Phys. Rev. E 60, 6803 (1999).

[27] T. Akizuki, K. Miyachi, Y. Takanishi, K. Ishikawa, H. Takezoe, and A. FukudaJpn. J. Appl. Phys., Part 1 38, 4832 (1999).

[28] F. Beaubois, J. P. Marcerou, H. T. Nguyen, and J. C. Rouillon, Eur. Phys. J. E 3, 273 (2000).

[29] P. M. Johnson, D. A. Olson, S. Pankratz, T. Nguyen, J. Goodby, M. Hird, and C. C. Huang, Phys. Rev. Lett. 84, 4870 (2000).

[30] L. S. Matkin, S. J. Watson, H. F. Gleeson, R. Pindak, J. Pitney, P. M. Johnson, C. C. Huang, P. Barois, A.-M. Levelut, G. Srajer, J. Pollmann, J. W. Goodby, and M. Hird, Phys. Rev. E 64, 021705 (2001).

[31] A. Cady, J. A. Pitney, R. Pindak, L. S. Matkin, S. J. Watson, H. F. Gleeson, P. Cluzeau, P. Barois, A.-M. Levelut, W. Galiebe, J. W. Goodby, M. Hird, and C. C. Huang, Phys. Rev. E 64, 050702(R) (2001).

[32] C. D. Cruz, J. C. Rouillon, J. P. Marcerou, N. Isaert, and H. T. Nguyen, Liq. Cryst. 28, 125 (2001).

[33] V. P. Panov, J. K. Vij, N. M. Shtykov, S. S. Seomun, D. D. Parghi, M. Hird, and J. W. Goodby, Phys. Rev. E 68, 021702 (2003).

[34] J. P. F. Lagerwall, P. Rudquist, and S. T. Lagerwall, Liq. Cryst. 30, 399 (2003)

[35] N. M. Shtykov, J. K. Vij, and H. T. Nguyen, Phys. Rev. E 63,
051708 (2001).

[36] N. M. Shtykov, J. K. Vij, R. A. Lewis, M. Hird, and J. W. Goodby, Liq. Cryst. 28, 1699 (2001).

[37] V. P. Panov, N. M. Shtykov, A. Fukuda, J. K. Vij, Y. Suzuki, R. A. Lewis, M. Hird, and J. W. Goodby, Phys. Rev. E 69, 060701(R) (2004).

[38] T. Isozaki, H. Takezoe, A. Fukuda, Y. Suzuki, and I. Kawamura, J. Mater. Chem. 4, 237 (1994).

[39] J. Hatano, Y. Hanakai, H. Furue, H. Uehara, S. Saito, and K. Murashiro, Jpn. J. Appl. Phys., Part 1 33, 5498 (1994).

[40] K. Itoh, M. Kabe, K. Miyachi, Y. Takanishi, K. Ishikawa, H. Takezoe, and A. Fukuda, J. Mater. Chem. 7, 407 (1997).

[41] Yu. P. Panarin, H. Xu, S. T. MacLughadha, J. K. Vij, A. J. Seed, M. Hird, and J. W. Goodby, J. Phys.: Condens. Matter 7, L351 (1995).

[42] Yu. P. Panarin, O. Kalinovskaya, J. K. Vij, and J. W. Goodby, Phys. Rev. E 55, 4345 (1997).

[43] A. V. Emelyanenko, A. Fukuda, and J. K. Vij (unpublished).

[44] M. Yamashita and S. Miyazima, Ferroelectrics 148, 1 (1993).

[45] J. Prost and R. Bruinsma, Ferroelectrics 148, 25 (1993).

[46] M. Cepic and B. Zeks, Mol. Cryst. Liq. Cryst. Sci. Technol., Sect. A 263, 61 (1995).

[47] M. Cepic and B. Zeks, Mol. Cryst. Liq. Cryst. Sci. Technol., Sect. A 301, 221 (1997).

[48] A. Roy and N. V. Madhusudana, Europhys. Lett. 41, 501 (1998).

[49] A. Roy and N. V. Madhusudana, Eur. Phys. J. E 1, 319 (2000).

[50] D. A. Olson, X. F. Han, A. Cady, and C. C. Huang, Phys. Rev. E 66, 021702 (2002).

[51] P. Bak, Phys. Today 39(12), 38 (1986).

[52] P. Bak and R. Bruinsma, Phys. Rev. Lett. 49, 249 (1982).

[53] R. Bruinsma and P. Bak, Phys. Rev. B 27, 5824 (1983).

[54] M. E. Fisher and W. Selke, Phys. Rev. Lett. 44, 1502 (1980).

[55] P. Bak and J. von Boehm, Phys. Rev. B 21, 5297 (1980).

[56] I. Musevic, M. Skarabot, G. Heppke, and H. T. Nguyen, Liq. Cryst. 29, 1565 (2002).

[57] M. Glogarova, H. Sverenyak, A. Fukuda, and H. Takezoe, Liq. Cryst. 14, 463 (1993).

[58] K. Miyachi, M. Kabe, K. Ishikawa, H. Takezoe, and A. Fukuda, Ferroelectrics 147, 147 (1993).

[59] V. Laux, N. Isaert, H. T. Nguyen, P. Cluzeau, and C. Destrade, Ferroelectrics 179, 25 (1996).

[60] V. Laux, N. Isaert, G. Joly, and H. T. Nguyen, Liq. Cryst. 26, 361 (1999).

[61] V. Laux, N. Isaert, V. Faye, and H. T. Nguyen, Liq. Cryst. 27, 81 (2000).

[62] P. M. Johnson, S. Pankratz, P. Mach, H. T. Nguyen, and C. C. Huang, Phys. Rev. Lett. 83, 4073 (1999).

[63] D. Schlauf, Ch. Bahr, and H. T. Nguyen, Phys. Rev. E 60, 6816 (1999).

[64] D. A. Olson, S. Pankratz, P. M. Johnson, A. Cady, H. T. Nguyen, and C. C. Huang, Phys. Rev. E 63, 061711 (2001).

[65] A. Cady, D. A. Olson, X. F. Han, H. T. Nguyen, and C. C. Huang, Phys. Rev. E 65, 030701(R) (2002).

[66] A. Cady, X. F. Han, D. A. Olson, H. Orihara, and C. C. Huang, Phys. Rev. Lett. 91, 125502 (2003).

[67] H. Orihara, A. Fajar, and V. Bourny, Phys. Rev. E 65, 040701(R) (2002).

[68] A. Fajar, H. Murai, and H. Orihara, Phys. Rev. E 65, 041704 (2002). 